ARTICLE

\title{
SNX13 reduction mediates heart failure through degradative sorting of apoptosis repressor with caspase recruitment domain
}

Jun $\mathrm{Li}^{1,2, \star}$, Changming $\mathrm{Li}^{1,3, \star}$, Dasheng Zhang ${ }^{1,2}$, Dan Shi ${ }^{1,2}$, Man $\mathrm{Qi}^{1,3}$, Jing Feng ${ }^{1,3}$, Tianyou Yuan ${ }^{1,2}$, Xinran $\mathrm{Xu}^{1,2}$, Dandan Liang ${ }^{1,2}$, Liang $\mathrm{Xu}^{1,2}$, Hong Zhang1,2, Yi Liu'1,2, Jinjin Chen ${ }^{1,3}$, Jiangchuan Ye ${ }^{1,3}$, Weifang Jiang ${ }^{4}$, Yingyu Cui ${ }^{1,5}$, Yangyang Zhang ${ }^{6}$, Luying Peng ${ }^{1,2,5}$, Zhaonian Zhou ${ }^{1,7} \&$ Yi-Han Chen ${ }^{1,2,3,5}$

Heart failure (HF) is associated with complicated molecular remodelling within cardiomyocytes; however, the mechanisms underlying this process remain unclear. Here we show that sorting nexin-13 (SNX13), a member of both the sorting nexin and the regulator of $G$ protein signalling (RGS) protein families, is a potent mediator of HF. Decreased levels of SNX13 are observed in failing hearts of humans and of experimental animals. SNX13-deficient zebrafish recapitulate HF with striking cardiomyocyte apoptosis. Mechanistically, a reduction in SNX13 expression facilitates the degradative sorting of apoptosis repressor with caspase recruitment domain (ARC), which is a multifunctional inhibitor of apoptosis. Consequently, the apoptotic pathway is activated, resulting in the loss of cardiac cells and the dampening of cardiac function. The N-terminal PXA structure of SNX13 is responsible for mediating the endosomal trafficking of ARC. Thus, this study reveals that SNX13 profoundly affects cardiac performance through the SNX13-PXA-ARC-caspase signalling pathway.

\footnotetext{
${ }^{1}$ Key Laboratory of Arrhythmias of the Ministry of Education of China, East Hospital, Tongji University School of Medicine, Shanghai 200120, China. ${ }^{2}$ Institute of Medical Genetics, Tongji University, Shanghai 200092, China. ${ }^{3}$ Department of Cardiology, East Hospital, Tongji University, Shanghai 200120, China.

${ }^{4}$ Institute of Neuroscience, Shanghai Institutes for Biological Sciences, Chinese Academy of Sciences, Shanghai 200031, China. ${ }^{5}$ Department of Pathology and Pathophysiology, Tongji University School of Medicine, Shanghai 200092, China. ${ }^{6}$ Cardiothoracic Surgical Department, the First Affiliated Hospital of Nanjing Medical University, Nanjing 210029, China. ${ }^{7}$ Laboratory of Hypoxic Cardiovascular Physiology, Shanghai Institutes for Biological Sciences, Chinese Academy of Sciences, Shanghai 200031, China. * These authors contributed equally to this work. Correspondence and requests for materials should be addressed to Y.-H.C. (email: yihanchen@tongji.edu.cn).
} 
eart failure (HF), the final stage of various cardiovascular diseases, is characterized by an impaired ability of the heart to fill with and/or eject blood, which is commensurate with the metabolic requirements of the body, resulting in the classic constellation of signs or symptoms of pulmonary and systemic venous congestion. HF affects $\sim 2 \%$ of the Western population; the incidence increases from $1 \%$ in 40 -year-old individuals to $10 \%$ of people above the age of 75 and is a leading cause of morbidity and mortality worldwide ${ }^{1}$. Despite major advances in the characterization of HF, many fundamental questions remain unanswered. Following long-standing insults, the heart undergoes protein and signal remodelling at the singlecell level in an attempt to maintain myocardial contractility and cardiac output. However, intracellular molecular remodelling may ultimately become maladaptive, leading to $\mathrm{HF}^{2}$. Little information is currently available regarding the molecular identity of the intracellular targeting pathways that underlie signal remodelling in failing cardiomyocytes.

It is clear that transmembrane proteins, lipids and other critical cargo molecules must be selectively sorted and transported between subcellular membrane-bound compartments via membrane trafficking processes. These endocytic compartments are also critical platforms for the regulation of cell signalling, which extends well beyond the traditional function of these compartments in controlling receptor degradation ${ }^{3}$. Mammalian phox-homology (PX) domain-containing molecules are predominantly found in the endosomal system, where these molecules regulate diverse trafficking and signalling processes in the cell ${ }^{4}$. Sorting nexins (SNXs) are a particular class of PX domain-containing proteins. Different SNXs may regulate the sorting/trafficking of various proteins and may couple endosomal sorting with specific signalling events ${ }^{5}$. In recent years, major progress has been made in understanding the biological function and relevance of SNXs in human diseases, including cancer, pathogen invasion, inflammation and Alzheimer's disease ${ }^{6}$. However, the role that SNXs play in the pathogenesis of HF remains unknown.

In this study, we find that SNX13 reduction profoundly affects HF development via a mechanism that involves the lysosomal degradation of apoptosis repressor with caspase recruitment domain (ARC) and the activation of pro-apoptotic caspases. These observations suggest that SNX13 plays a crucial role in preserving cardiomyocyte survival and cardiac performance by targeting the endosomal sorting of anti-apoptotic cytoprotective signals.

\section{Results}

Endogenous SNX13 is downregulated in failing hearts. To explore the potential role of SNX family molecules in HF, we performed an mRNA screen for SNX genes that might experience changes in end-stage failing human hearts. On the basis of a more profound reduction compared with all 33 SNX isoforms screened, we identified $S N X 13$ as a candidate regulator of HF development (Fig. 1a). Next, we analysed the expression of SNX13 in a mouse model of HF, in which the mice underwent transverse aortic constriction or sham surgery. As shown in Fig. 1b,c, myocardial SNX13 mRNA and protein levels were clearly reduced in the mouse model of HF compared with the sham-operated control mice. These results suggested a correlation between SNX13 reduction and $\mathrm{HF}$.

Loss of SNX13 in zebrafish causes severe HF. The reduction in SNX13 protein expression during HF development suggested a potential functional role for SNX13 in this process. To test this hypothesis, we examined the effect of SNX13 downregulation on cardiac function; however, SNX13 ablation is embryonically lethal

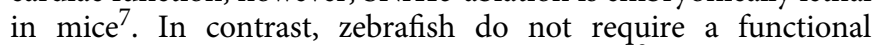
circulatory system during early development ${ }^{8}$; thus, we used zebrafish embryos to evaluate whether in vivo SNX13 loss could cause cardiac dysfunction. The zebrafish orthologue of mammalian SNX13 was first identified by performing a BLAST search of the zebrafish Ensembl database. The zebrafish SNX13 gene encoded a protein with a high degree of identity to the human, mouse and rat SNX13 proteins (Fig. 2a). Next, we determined the expression of SNX13 mRNA in zebrafish embryos using whole-mount antisense RNA in situ hybridization. We found that SNX13 transcripts could be detected in the hearts of zebrafish embryos at $72 \mathrm{~h}$ post fertilization (h.p.f.; Fig. 2b). The SNX13 transcript was also expressed in heart, liver, brain and skeletal muscle tissues in adult zebrafish (Supplementary Fig. 1).

To inactivate SNX13 function, morpholino-modified antisense oligonucleotides directed against either the translational start site (MO-SNX13) or the splice donor sites of exon 2 (MO-SNX13 ${ }^{s p l}$ ) were injected into one-cell-stage zebrafish embryos. We detected an aberrant splice product in MO-SNX13 $3^{s p l}$-injected embryos, whereas wild-type SNX13 mRNA was significantly reduced (Fig. 2c). A western blot analysis confirmed the effective knockdown of the SNX13 protein using MO-SNX13 or MOSNX13 ${ }^{\text {spl }}$ (Fig. 2c).

We observed that MO-SNX13- $\left(8 \mathrm{ng} \mathrm{nl}^{-1}\right)$ or MO-SNX13 ${ }^{\text {spl }}$ injected ( $4 \mathrm{ng} \mathrm{nl}^{-1}$ ) embryos showed normal heart morphogenesis during the first 24 h.p.f. Between 48 and 72 h.p.f., $65 \%$ of the MO-SNX13- $(n=102)$ and $57 \%$ of the MO-SNX13 ${ }^{s p l}$-injected $(n=95)$ zebrafish embryos began to develop severe HF. The injection of a mismatch morpholino (MO-Ctrl) did not lead to the MO-SNX13-specific cardiac phenotype $(n=110$; data not shown). Then, we determined when HF became evident in these embryos. Compared with the MO-Ctrl siblings, these morphants demonstrated dilated atrial and ventricular chambers (Fig. 2d) with a decreased systolic function, as demonstrated by haematoxylin and eosin (H\&E) staining of heart sections (Fig. 2e), by stroke volume (SV) and by cardiac output (Fig. $2 \mathrm{f}$ ). The atria of MO-SNX13- and MO-SNX13 ${ }^{s p l}$-injected zebrafish were also severely dilated with blood reflux from the atrium to the sinus venosus, which is another feature of congestive HF (Supplementary Movie 1).

Apoptosis of cardiomyocytes underlies SNX13-deficient HF. To evaluate how SNX13 deficiency led to HF in the morphant embryos, we first analysed whether the disturbed structure of cardiac muscle cells was responsible. Notably, cellular autolysis, which is a form of programmed cell death, was observed in SNX13-deficient zebrafish hearts. Autolysis became more pronounced as the SNX13-deficient embryos developed beyond 72 h.p.f. (Fig. 3a), suggesting that cardiomyocyte death played an essential role in SNX13 deficiency-induced HF.

Next, we used an acridine orange (AO) staining assay to indicate cells undergoing apoptosis. We compared cell death in the developing hearts of MO-SNX13-injected and scrambleinjected (MO-Ctrl) embryos at 48 h.p.f. Increased numbers of AO-positive cardiomyocytes were found in MO-SNX13-injected embryos. In contrast, no cell death was detected in the atria or ventricles of scramble-injected embryos (Fig. 3b; Supplementary Movie 2). In another set of experiments, we also adopted a transgenic zebrafish line expressing the cardiac-specific myosin light chain-2 gene fused to green fluorescence protein (GFP; cmlc2:GFP) in combination with the staining of cleaved caspase-3 to show the apoptotic cardiomyocytes in the embryonic hearts. We found that the positive caspase- 3 signals were observed in the cardiomyocytes of morphants but not of MO-Ctrl siblings 

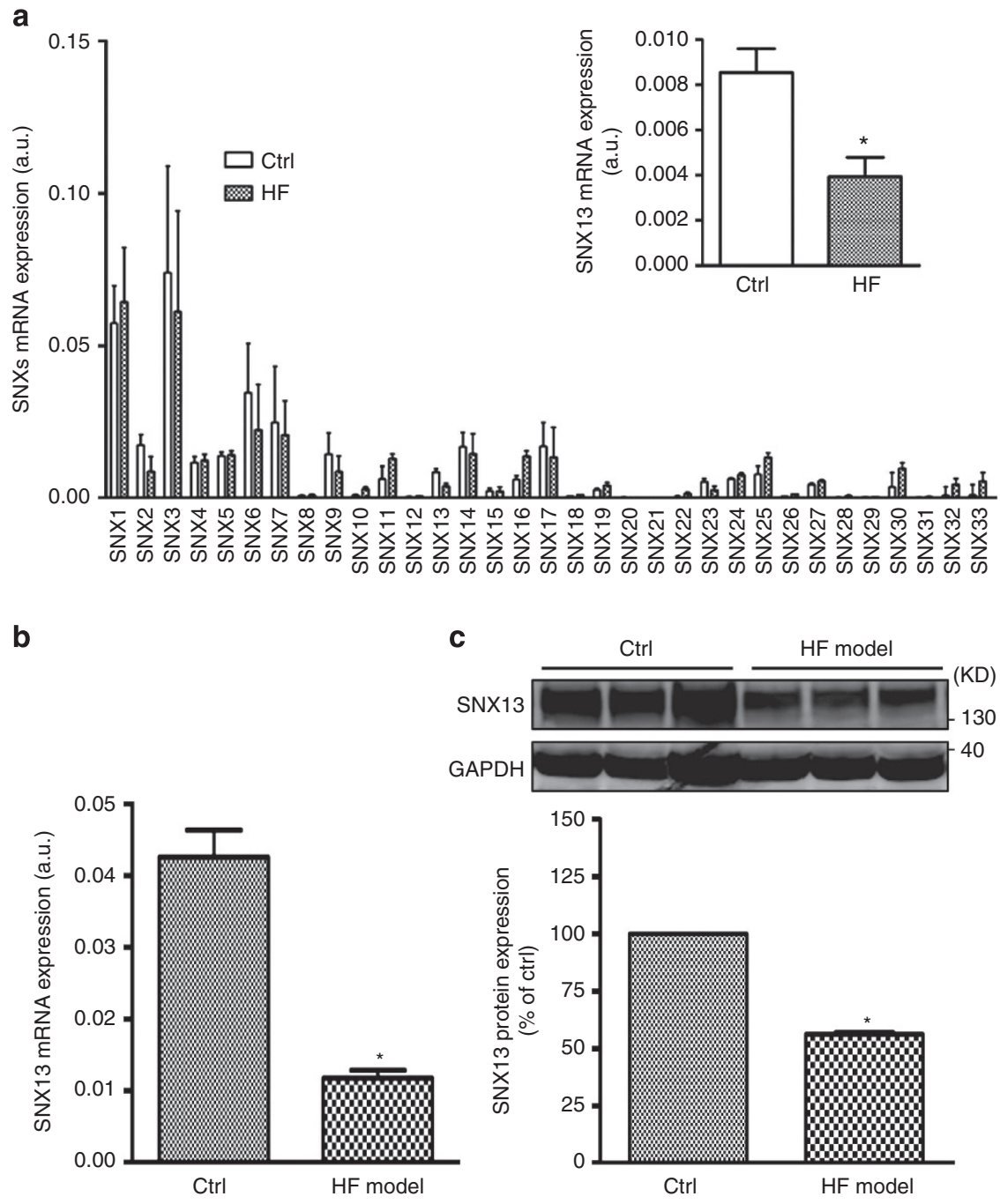

Figure 1 | SNX13 expression is reduced in failing hearts. (a) A quantitative PCR-based mRNA expression screen for all known SNX isoforms in end-stage failing heart tissues ( $N=5$ per HF, $N=3$ per healthy control). A significant reduction in SNX13 mRNA expression was identified from all 33 SNX isoforms. (b) SNX13 mRNA expression in failing cardiac tissues from experimental HF mice. (c) Western blot analysis of SNX13 protein expression in failing mice hearts $\left(N=5\right.$ per TAC-induced $\mathrm{HF}, N=3$ per sham-operated control). Ctrl, control. Data are means \pm s.e.m. ${ }^{\star} P<0.05$ compared with $\mathrm{Ctrl}$, by one-tailed unpaired Student's $t$-test with Welch's correction.

(Supplementary Fig. 2). These findings indicated that SNX13 reduction induced significant apoptosis of cardiomyocytes.

Given the induction of apoptosis in SNX13-deficient zebrafish cardiomyocytes, we hypothesized that SNX13 reduction might also trigger similar events in primary mammalian cardiomyocytes. Small interfering RNA (siRNA) was used to reduce SNX13 levels in neonatal rat ventricular myocytes (NRVMs). Compared with mock control or SNX13 overexpression, the knockdown of SNX13 was sufficient to drive apoptosis, as demonstrated by DNA laddering and by fluorescence-activated cell sorting assays (Fig. 3c,d). These data indicated a conserved role for SNX13 in the control of cardiac cell death and survival.

Extrinsic apoptosis contributes to SNX13-deficient HF. To explore the effect of SNX13 deficiency-induced cardiomyocyte apoptosis on HF predisposition, we subjected wild-type and SNX13-deficient zebrafish embryos to chemical compounds known to exert antagonistic effects on critical apoptotic molecules. We determined the cardiac function after inhibiting caspase-8 (a key mediator of extrinsic apoptosis), caspase-9 (an important mediator of intrinsic apoptosis) and caspase-3 (a terminal effector for the intrinsic and extrinsic apoptotic pathways) ${ }^{9}$. Caspase-8 and caspase-3 inhibition markedly counteracted the cardiac cell apoptosis induced by SNX13 knockdown, whereas caspase-9 inhibition conferred a weaker effect (Fig. 4a,b; Supplementary Movie 3). Correspondingly, morphants with inhibited caspase- 8 or caspase- 3 underwent significant improvements in SV and in cardiac output compared with morphants with caspase-9 inhibition (Fig. 4c).

We further examined whether SNX13 downregulation activated apoptotic signals in NRVMs. Western blot analysis was used to examine the expression levels of procaspases (inactivated caspases) and of cleaved caspases (activated caspases). As shown in Fig. $4 \mathrm{~d}$, cleaved caspase- 8 and caspase- 3 were elevated in NRVMs with SNX13 knockdown, whereas no significant changes in the expression of procaspase-8 and procaspase-3 were observed. We also analysed the expression of molecules that are critical to the intrinsic apoptotic pathway, including caspase-9, cytochrome $c, \mathrm{Bcl} 2$ and Bax. Compared with the positive control with $\mathrm{H}_{2} \mathrm{O}_{2}$ treatment $(150 \mu \mathrm{M}, 6 \mathrm{~h})$, SNX13 reduction did not affect the expression of pro- and cleaved caspase-9, Bcl2 or Bax 
a

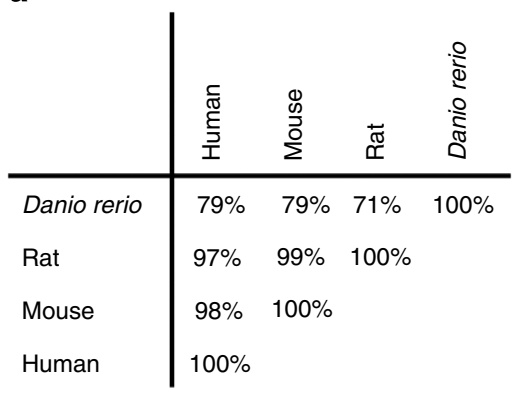

b

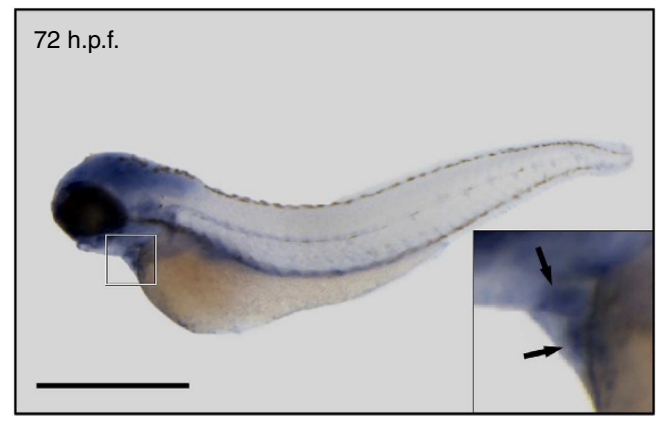

d

MO-Ctrl

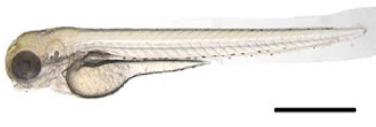

MO-SNX13

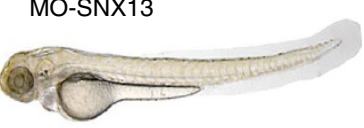

GAPDH

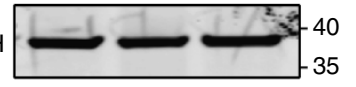

MO- $\quad \mathrm{MO}-$

ctrl SNX13spl
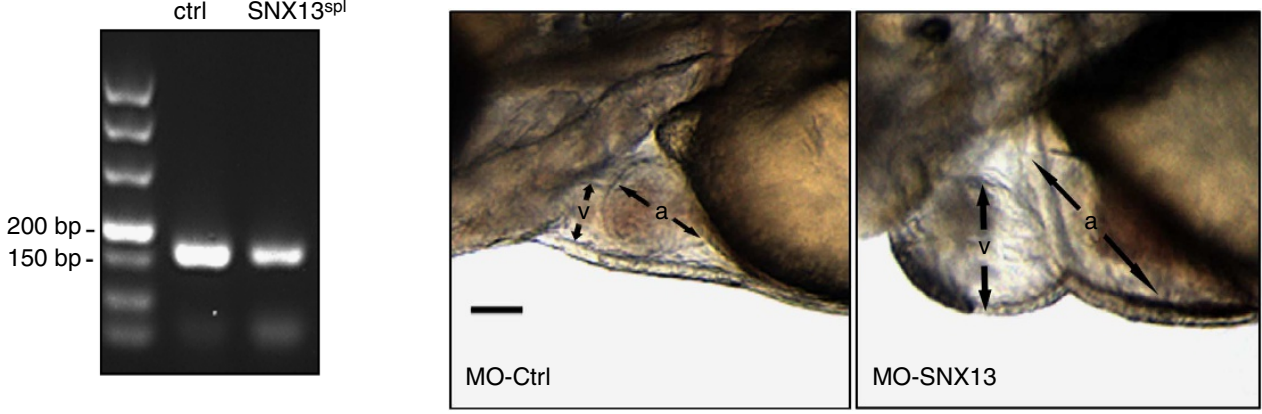

e
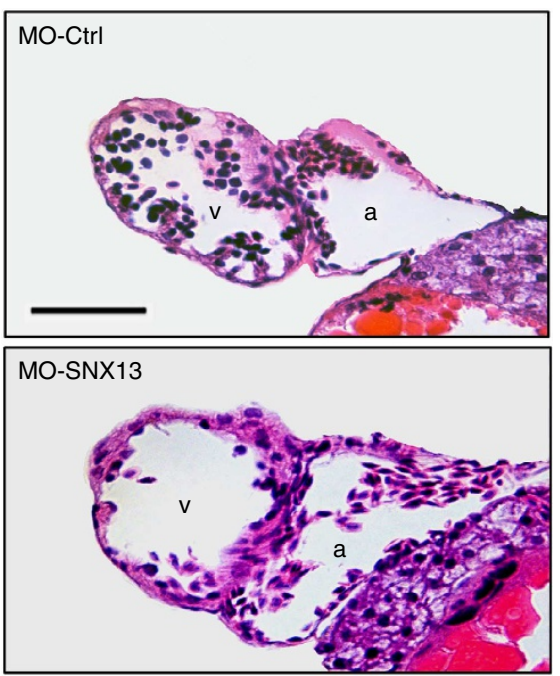

$\mathbf{f}$
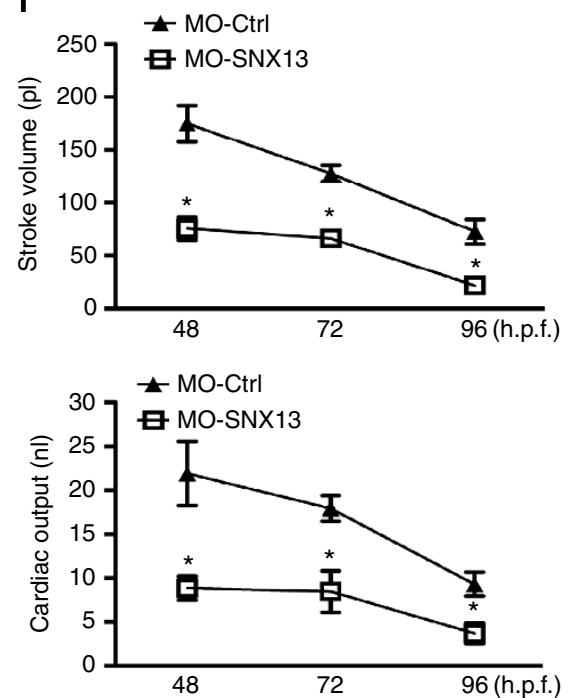

Figure 2 | SNX13-deficient zebrafish hearts display severe dysfunction. (a) Zebrafish SNX13 has high sequence identity with mammalian orthologues across the entire peptide sequence. (b) In situ hybridization of SNX13 in the zebrafish embryos at $72 \mathrm{~h}$ post fertilization (h.p.f.). The rectangular box indicates the heart region. Scale bar, $500 \mu \mathrm{m}$. (c) Examination of SNX13 knockdown in zebrafish embryos. Upper, western blot analysis of the SNX13 protein level in MO-Ctrl-, MO-SNX13 spl- and MO-SNX13-injected embryos. Glyceraldehyde 3-phosphate dehydrogenase (GAPDH) is used as the loading control.

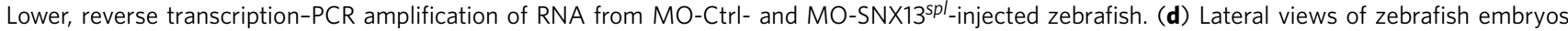
at 72 h.p.f. Scale bar, $500 \mu \mathrm{m}$. Bottom, lateral close-up view of zebrafish hearts. The ventricular (v) and atrial (a) chambers of MO-SNX13-injected zebrafish are dilated. Scale bar, $40 \mu \mathrm{m}$. (e) H\&E staining of zebrafish heart sections of MO-Ctrl- and MO-SNX13-injected embryos at 72 h.p.f. Scale bar, $50 \mu \mathrm{m}$. (f) Analysis of the stroke volume and cardiac output using high-speed laser confocal line scanning. Ctrl, control. $N=32$ per morphant, $N=26$ per $\mathrm{MO}-\mathrm{Ctrl}$. Data are means \pm s.e.m. ${ }^{\star} P<0.05$ compared with MO-Ctrl, by one-way analysis of variance with Bonferroni's post hoc test. 
a

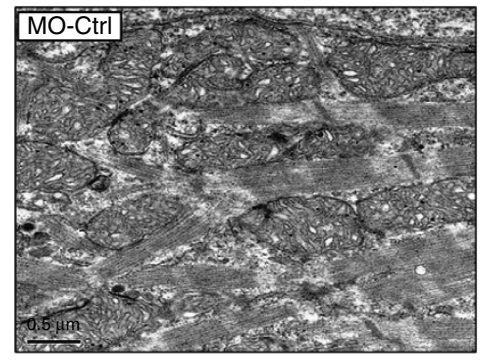

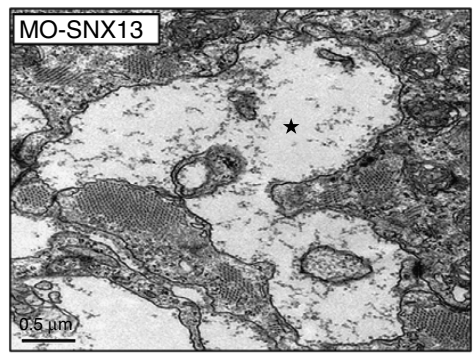

b
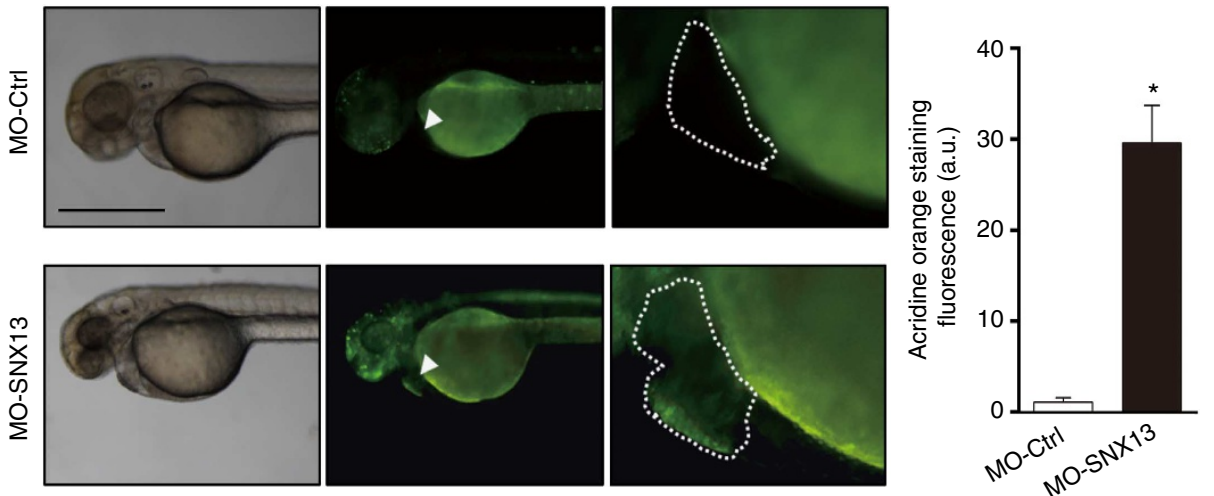

C

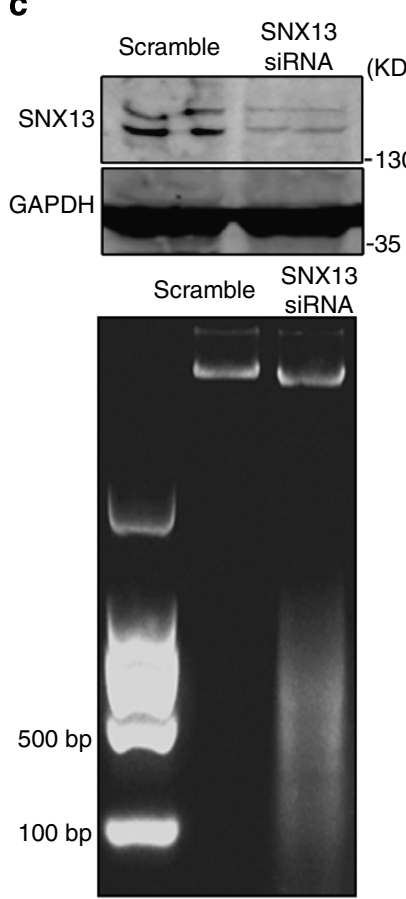

d
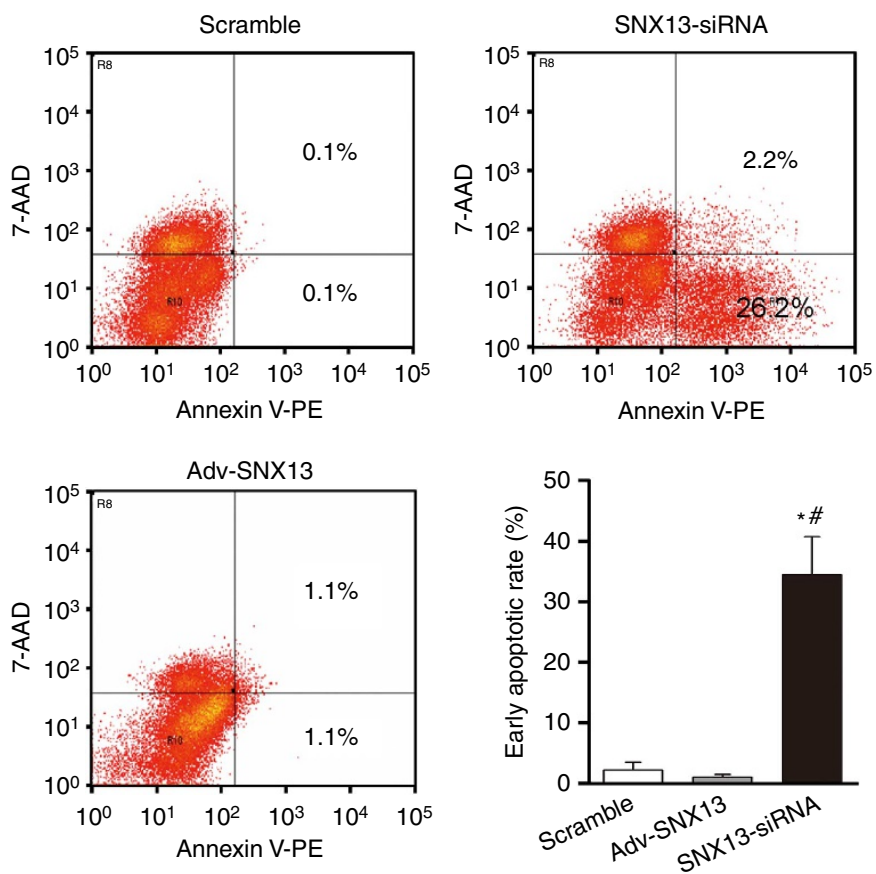

Figure 3 | SNX13 deficiency induces cardiac autolysis and apoptosis. (a) Transmission electron microscopy of zebrafish embryonic hearts at 72 h.p.f. Overt cardiomyocyte autolysis (black star) was observed in morphant ventricular myocytes. Scale bar, $0.5 \mu \mathrm{m}$. (b) AO staining of zebrafish embryos demonstrated cardiomyocyte apoptosis in SNX13 morphant hearts at 48 h.p.f. The white arrowhead and dashed line indicate the cardiac region. Right: pooled data for the $A O$ fluorescence intensity in the cardiac area. $N=6$ per morphant, $N=6$ per MO-Ctrl. Scale bar, $400 \mu \mathrm{m}$. Data are means \pm s.e.m. ${ }^{\star} P<0.05$ compared with MO-Ctrl, by one-tailed unpaired Student's $t$-test with Welch's correction. (c) A DNA laddering assay in SNX13 knockdown NRVMs. Top, western blot analysis for SNX13 at $48 \mathrm{~h}$ after transfection with siRNA. (d) Apoptosis analysis using flow cytometry after Annexin $V$ PE/7-AAD staining. Adv-SNX13, adenovirus-mediated SNX13 overexpression. The flow cytometry profile of a representative experiment is shown. The data were gated for damaged cells (Annexin $\vee \mathrm{PE}^{-}$and 7-AAD ${ }^{+}$, top left quadrant), late apoptotic cells (Annexin $\vee \mathrm{PE}^{+}$and $7-\mathrm{AAD}^{+}$, top right quadrant), viable cells (Annexin $\vee \mathrm{PE}^{-}$and 7- $A A D^{-}$, bottom left quadrant) and for early apoptotic cells (Annexin $\vee \mathrm{PE}^{+}$and 7-AAD ${ }^{-}$, bottom right quadrant). The percentage of early apoptotic cells was determined using flow cytometry. Ctrl, control; SNX13-wt, wild-type SNX13 overexpression. $n=3$. Data are means \pm s.e.m. ${ }^{\star} P<0.05$ compared with scramble, $\# P<0.05$ compared with groups other than scramble, by one-way analysis of variance with Bonferroni's post hoc test. $N$ represents the number of animals, and $n$ represents the number of experiments. 
a

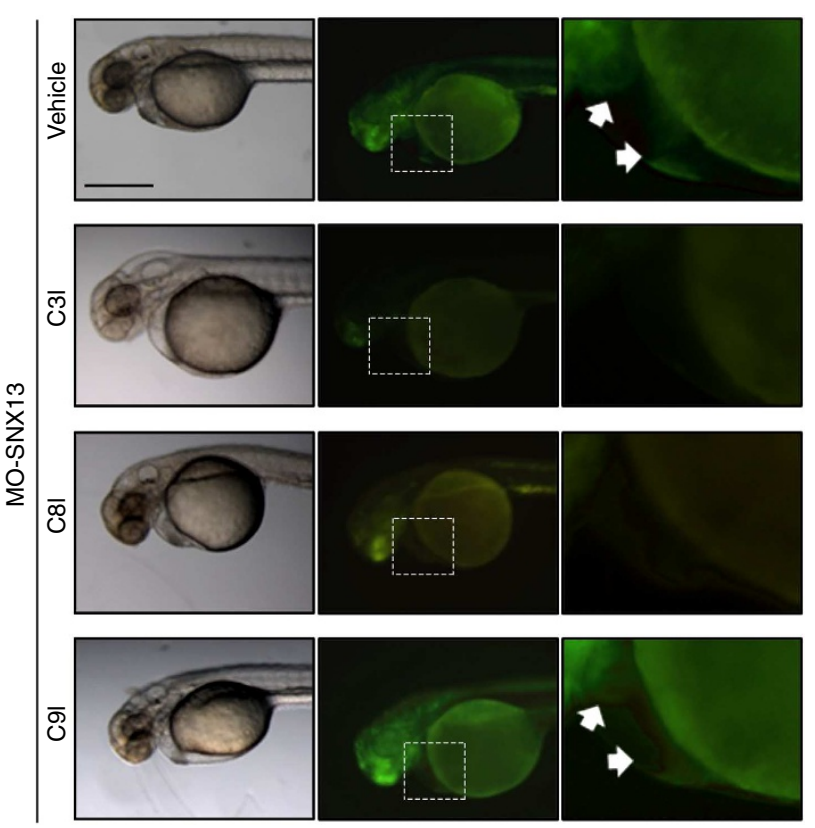

b

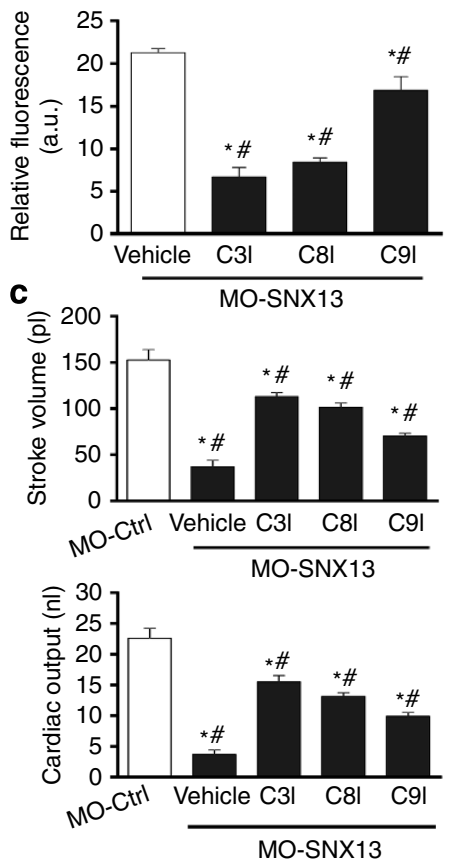

d
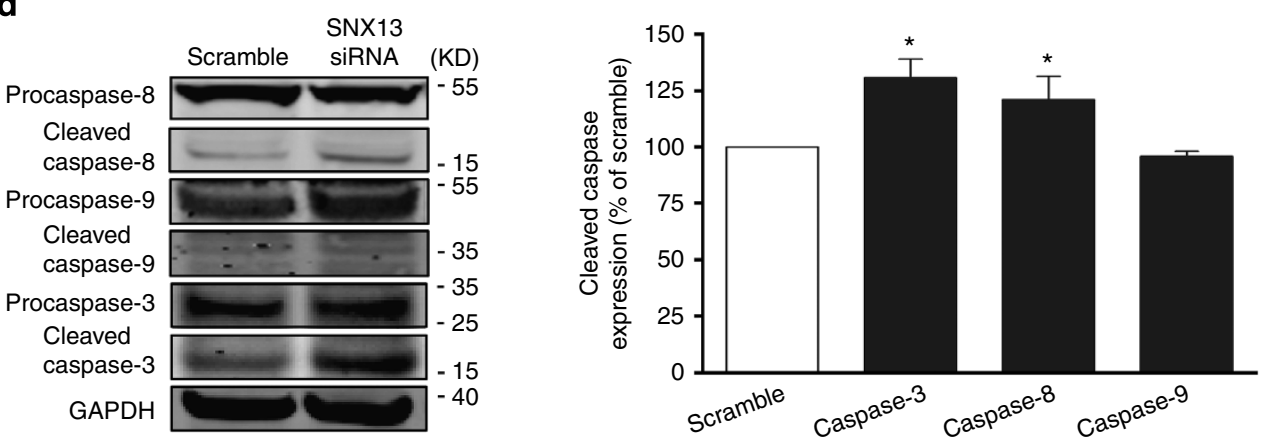

e SNX13 $\quad \mathrm{H}_{2} \mathrm{O}_{2}$
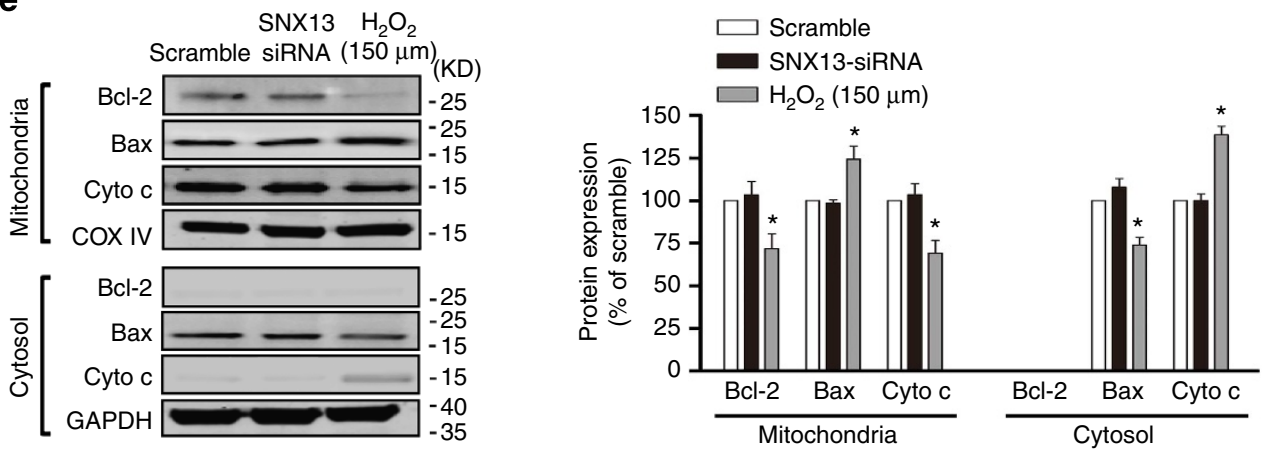

Figure 4 | SNX13-deficent HF links to extrinsic cardiomyocyte apoptosis. (a-c) The effects of caspase-specific inhibitory compounds (caspase-3 inhibitor Ac-DEVD-CHO, $30 \mu \mathrm{M}$; caspase-8 inhibitor Z-IETD-FMK, $10 \mu \mathrm{M}$; and caspase-9 inhibitor Z-LEHD-FMK, $20 \mu \mathrm{M}$ ) on cardiac apoptosis (a,b) and the stroke volume and cardiac output at 48 h.p.f. (c). The AO fluorescent intensity was used to evaluate apoptotic cardiomyocytes in the cardiac area (defined by arrows and outlined by dashed lines) (b). $N=8$ per morphant, $N=10$ per $\mathrm{MO}$ control. C $3 \mathrm{I}, \mathrm{C} 8 \mathrm{I}$ and C9I represent inhibitors of caspase-3, -8 and -9 , respectively. Scale bar, $400 \mu \mathrm{m}$. (d) Western blot analysis of the critical molecules of the extrinsic and intrinsic apoptotic pathways. SNX13 knockdown in NRVMs increased caspase-3 and caspase-8 expression but did not affect caspase- 9 expression, as demonstrated by representative blots. Left, typical western blots; right, pooled data. (e) Western blot analysis of the protein levels of cytochrome $c$, Bcl2 and Bax in the cytosolic and mitochondrial fractions of SNX13-deficient NRVMs. Left, typical western blots; right, pooled data. Ctrl, control. $n=3$. Data are means \pm s.e.m. ${ }^{\star} P<0.05$ compared with Ctrl; \#P<0.05 compared with groups other than Ctrl, by one-way analysis of variance with Bonferroni's post hoc test. $N$ represents the number of animals, and $n$ represents the number of experiments. 
(Fig. 4e; Supplementary Fig. 3). In addition, cytochrome $c$ was not detected in cytosolic fractions of SNX13-defective NRVMs. Taken together, the SNX13 knockdown-mediated cardiomyocyte apoptosis is primarily attributed to the activation of the extrinsic apoptotic pathway.

SNX13 regulates ARC-mediated cardiomyocyte apoptosis. As described in the previous section, caspase- 8 is a crucial signalling molecule in the extrinsic apoptotic pathway and is activated in SNX13-deficient cardiomyocytes. ARC, which is specifically expressed in striated muscles and in neurons, is a master regulator of cardiac death signalling. This molecule binds to caspase8 , inhibits its activity ${ }^{10, P 1}$ and provides cardioprotective effects during HF development ${ }^{12}$. Thus, next, we investigated whether SNX13 affects caspase- 8 activity by modulating the anti-apoptotic protein ARC.

We performed an expression analysis of ARC in the failing hearts of experimental HF mice and in SNX13-deficient NRVMs. A significantly reduced level of the ARC protein was observed in these cells (Fig. 5a,b). To explore the underlying mechanism for the decreased expression of ARC in HF, ARC mRNA levels were determined using real-time PCR. The ARC mRNA levels did not differ between mouse HF hearts and related controls (Supplementary Fig. 4a) or between SNX13-deficient NRVMs and normal cardiomyocytes (Supplementary Fig. 4b), suggesting that the regulation of ARC expression occurs at the posttranscriptional level. Given the protein sorting/trafficking properties of SNX13, we investigated whether the loss of SNX13 directed ARC movement towards lysosomes and, thus, degradation. We found that the specific proteasome/lysosome inhibitor bafilomycin A1 $(100 \mathrm{nM})^{13}$ counteracted the ARC degradation that resulted from decreased SNX13 levels in NRVMs (Fig. 5c), but that proteasome inhibition with MG132 $(0.5 \mu \mathrm{M})^{14}$ did not affect ARC expression in the SNX13-deficient cardiomyocytes (Fig. 5d; Supplementary Fig. 5). In addition, RNA interference specific for LAMP1 was adopted to impair the lysosomal degradation pathway. LAMP1 depletion significantly reconciled the ARC reduction induced by SNX13 knockdown (Fig. 5e), which was similar to the effects of bafilomycin A1. Notably, the overexpression of ARC markedly rescued the SNX13 reductioninduced apoptosis of NRVMs (Fig. 5f) and the SNX13-deficient cardiac failure (Supplementary Fig. 6), suggesting that SNX13 deficiency-associated apoptosis was involved in the reduced ARC levels. Taken together, these results demonstrate that SNX13 directly modulates ARC stability and that SNX13 reduction mediates the apoptotic death of cardiomyocytes by promoting ARC degradation.

PXA structure of SNX13 is responsible for ARC degradation. To further elucidate the SNX13-dependent regulation of ARC stability in cardiomyocytes, we analysed the potential interaction of SNX13 with ARC and tracked the intracellular trafficking of ARC under SNX13 reduction in $\mathrm{H} 9 \mathrm{C} 2$ cells, which is a permanent cell line derived from rat cardiac tissue. First, the association of SNX13 with ARC was tested using co-immunoprecipitation. Proteins in lysates from freshly isolated intact rat heart tissues were immunoprecipitated with a specific antibody against SNX13 or against ARC. SNX13 was found to co-precipitate with ARC and vice versa (Fig. 6a). Then, we performed immunofluorescence labelling to show the subcellular locations of SNX13 and of ARC. We observed that the endogenous SNX13 and ARC localized to early endosomes, as demonstrated by the co-localization of SNX13 and of ARC with EEA1 (a marker of early endosomes; Fig. 6b). Identification of their expression in the purified early endosomes (Fig. 6c) further supported a physical interaction between SNX13 and ARC. Next, the SNX13-mediated endosomal trafficking of ARC was studied. Staining with an anti-SNX13 antibody was employed to indicate the cells with SNX13 knockdown. We found that most ARC proteins co-localized with LysoTracker (an indicator of lysosomes) in SNX13-deficient cells at $72 \mathrm{~h}$ after transfection with SNX13-siRNA (Fig. 6d). The biochemical and fluorescence microscopic data suggest that SNX13 directly interacts with ARC in the endosomes and that a reduction in SNX13 expression may facilitate the lysosomal targeting of ARC.

To gain a detailed understanding of the structural basis for the SNX13-mediated endosomal sorting of ARC, we further examined several truncated versions of SNX13 for their ability to regulate the intracellular trafficking of ARC. First, we deleted the amino-terminal PXA domain (residues 97-284), PX domain (residues 563-676), G protein signalling (RGS) domain (373-513) or carboxy-terminal PX-associated domain (PXC; 779-808; Fig. 7a). Next, we analysed the potential interaction of ARC with these Flag-tagged mutant fusion proteins using co-immunoprecipitation. As shown in Fig. 7b, among the four truncated mutants, only the PXA-truncated mutant ( $\triangle$ PXA-SNX13) could not co-immunoprecipitate with ARC, suggesting that the PXA domain may be critically responsible for the interaction between ARC and SNX13. Then, we employed a cellular imaging technique to demonstrate the subcellular localization of ARC in cells overexpressing these mutants. The mutants were transiently overexpressed in cells, and an anti-flag antibody was used to show the cells expressing these mutants (Fig. 7c). Immunofluorescence imaging showed that most of the tagged truncated proteins were not correctly localized to endosomes, which is different from endogenous proteins. SNX13 mutant with the N-terminal PXA domain deletion induced the lysosomal trafficking of ARC, whereas the other three truncated mutants did not affect the subcellular localization of ARC proteins (Fig. 7c,d), mimicking the downregulation of full-length SNX13 and suggesting that a small amount of the expressed proteins retain activity and induce the cellular phenotypes in ARC localization. Finally, we evaluated whether the overexpression of these mutants affected the activation of caspase-8, which is the direct target of ARC. Western blot examination demonstrated that $\triangle$ PXA-SNX13 overexpression increased the expression of the cleaved caspase8 and reduced the ARC expression, as observed by SNX13 knockdown (Fig. 7e,f). These results indicated that the N-terminal PXA domain structure may be the decisive module for the SNX13 reduction-mediated degradative sorting of the ARC protein and for caspase activation.

\section{Discussion}

Our study reveals that SNX13 regulates cardiomyocyte fate and cardiac performance via its N-terminal PXA domain-mediated endosomal trafficking of ARC. Five major findings that we obtained from observational clinics and from relevant experimental models support this conclusion. First, endogenous SNX13 is significantly reduced in the failing hearts of several species. Second, SNX13-deficient model organisms exhibit a striking decrease in in vivo cardiac systolic function, which is primarily linked to extrinsic apoptotic cardiomyocyte death. Third, the inhibition of cardiomyocyte apoptosis largely rescues the cardiac dysfunction associated with SNX13 reduction. Fourth, SNX13 reduction facilitates the degradation of ARC, and ARC overexpression counteracts the apoptotic death of cardiomyocytes. Fifth, the N-terminal PXA structure of SNX13 mediates the endosomal trafficking of ARC.

At present, 33 mammalian SNXs have been identified. Dr Farquhar's group was the first one to describe the SNX13/ RGS-PX1 bifunctional role in the regulation of G-protein $(\mathrm{G} \alpha \mathrm{s})$ 
a
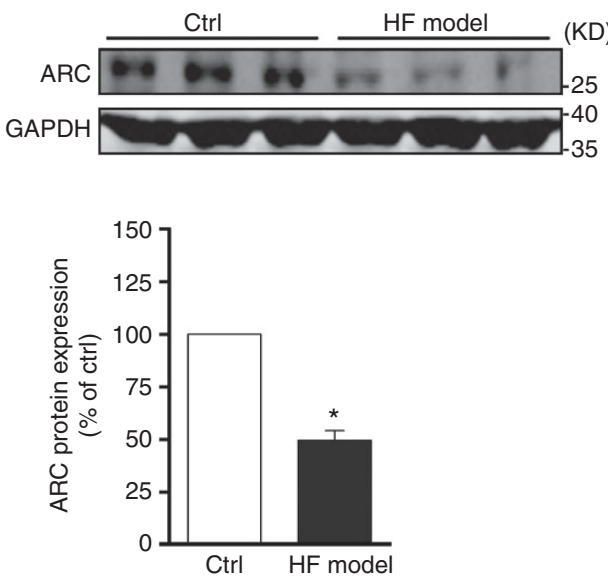

C
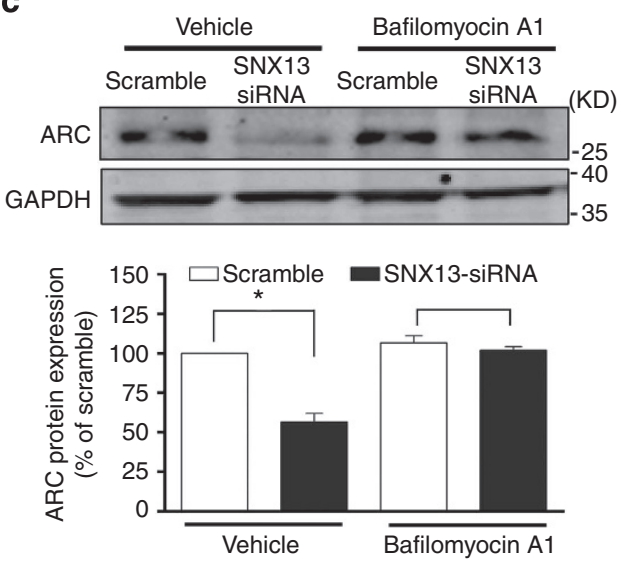

e
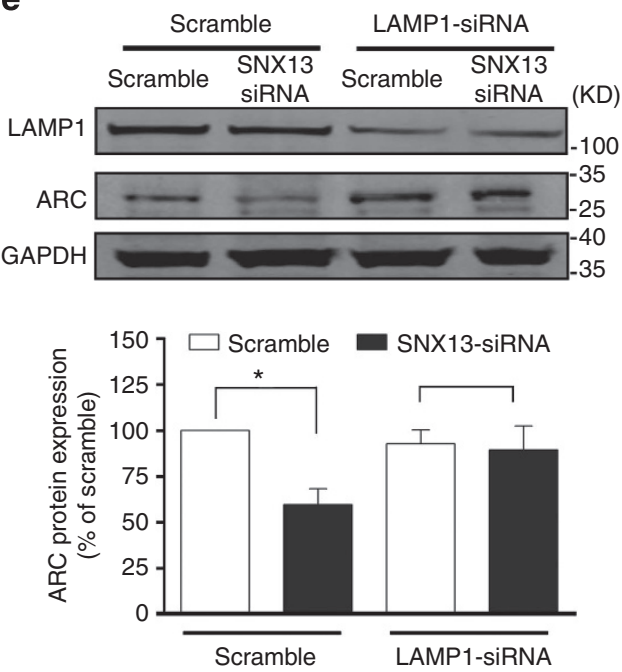

b
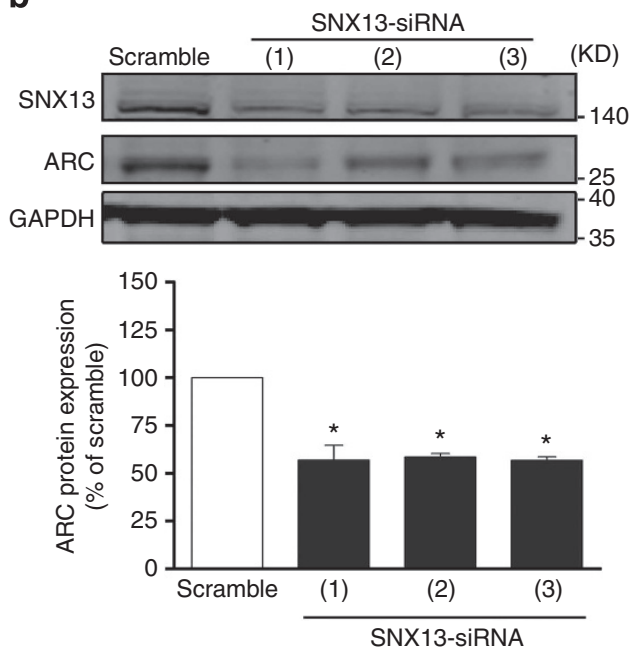

d
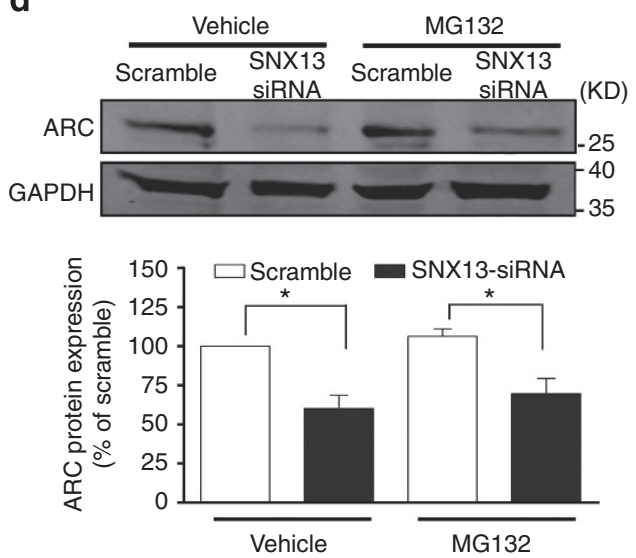

f
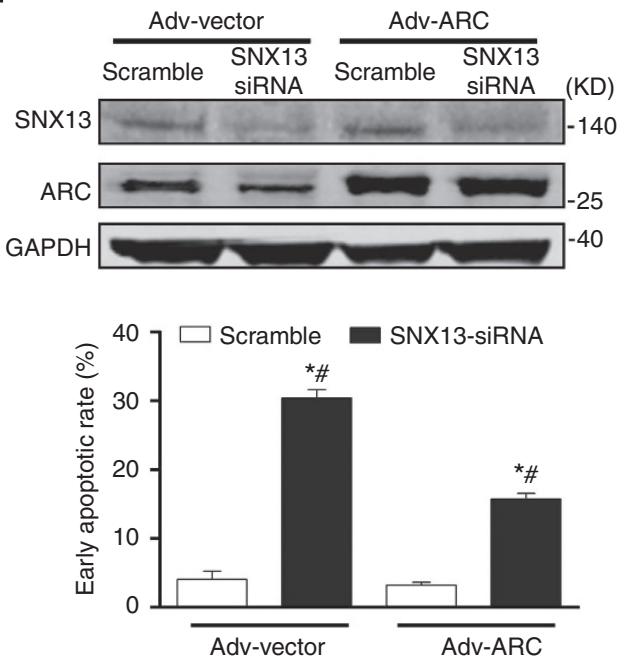

Figure 5 | ARC degradation mediates SNX13-deficient myocyte apoptosis. (a,b) ARC protein expression levels in failing mouse hearts and in SNX13-deficient NRVMs. $n=3$. Data are means \pm s.e.m. ${ }^{\star} P<0.05$ compared with scramble, by one-way analysis of variance (ANOVA) with Bonferroni's post hoc test. (c,d) Western blot analysis of ARC in SNX13-deficient NRVMs in the presence or absence of the lysosomal inhibitor bafilomycin A1 (Ba-A1, $100 \mathrm{nM})$ and the proteasome inhibitor MG132 $(0.5 \mu \mathrm{M})$. Top, representative blots; bottom, quantified data. Ctrl, control. $n=3$. ${ }^{\star} P<0.05$ compared with Ctrl or mock. (e) Effects of LAMP1 deletion on the ARC expression in SNX13-deficient cells. Top, typical western blots; bottom, quantified data.

(f) Cardiomyocyte apoptosis analysis using flow cytometry after Annexin V PE/7-AAD staining. Top, western blot analysis of ARC and SNX13 proteins in NRVMs; bottom, pooled data for the apoptotic rate of NRVMs. Data are means \pm s.e.m. ${ }^{\star} P<0.05$ compared with scramble, \#P<0.05 compared with groups other than scramble, by one-way ANOVA with Bonferroni's post hoc test. All of the data were derived from three separate experiments. $n$ represents the number of experiments. 
a

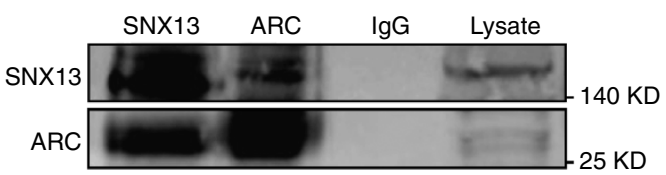

b
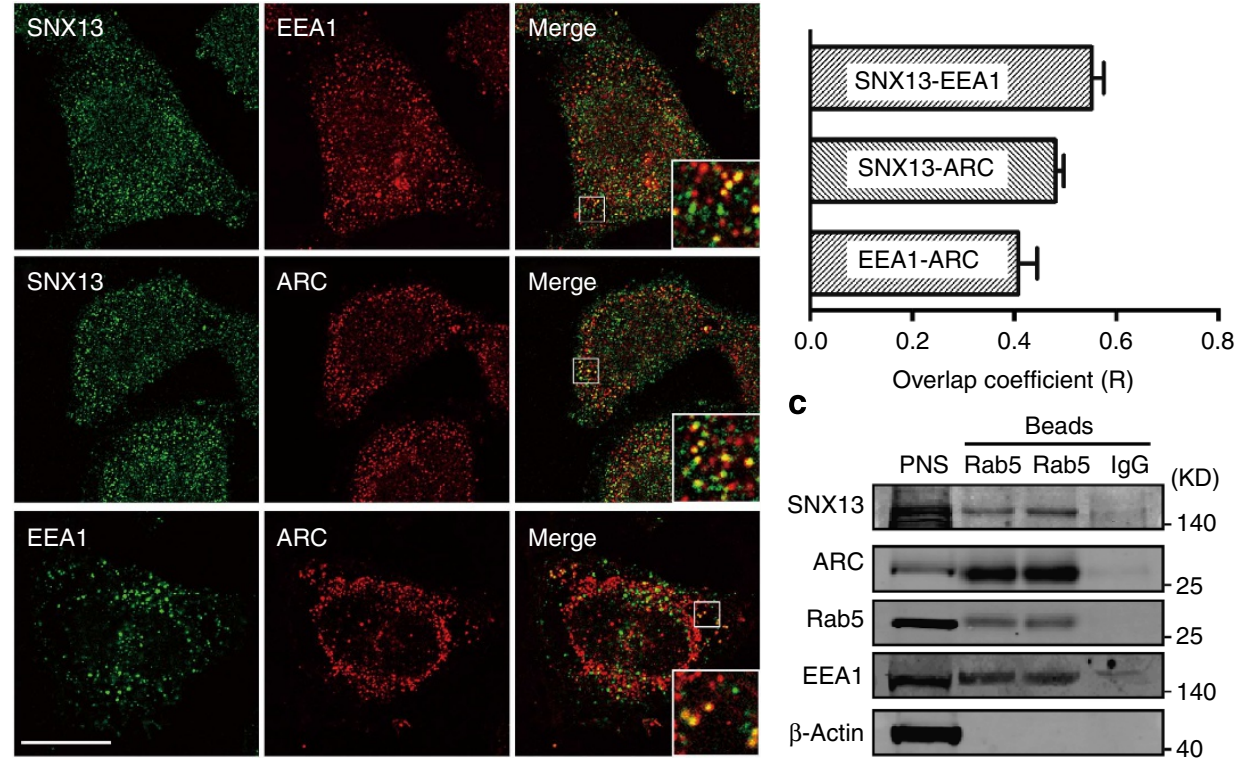

C

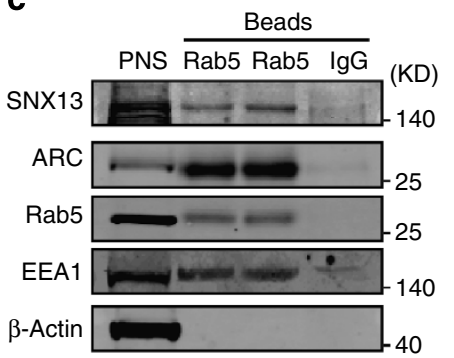

d
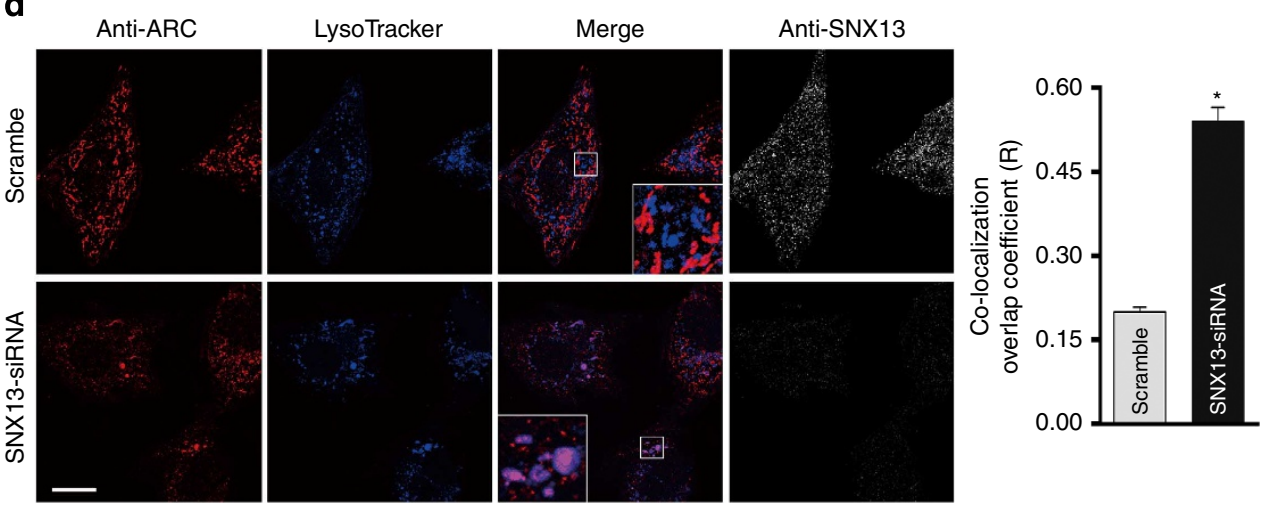

Figure 6 | SNX13 deficiency induces the lysosomal translocation of ARC. (a) Co-immunoprecipitation of SNX13 with ARC from NRVM lysates. (b) A representative confocal fluorescence microscopy image of the co-localization of EEA1/SNX13, EEA1/ARC and of SNX13/ARC in normal H9C2 cardiac cells. Right, pooled data showing the overlap coefficient. Scale bar, $20 \mu \mathrm{m}$. (c) Western blot examination of SNX13 and ARC proteins in the purified early endosomes from NRVMs. PNS, postnuclear supernatant. The representative blots were derived from three separate experiments. (d) LysoTracker and ARC images in $\mathrm{H} 9 \mathrm{C} 2$ cells with SNX13 knockdown. Right, pooled data showing the overlap coefficient. The representative images were derived from three separate experiments. Scale bar, $25 \mu \mathrm{m}$. Data are means \pm s.e.m. ${ }^{\star} P<0.05$ compared with scramble, by one-tailed unpaired Student's $t$-test with Welch's correction.

signalling and of endosomal sorting ${ }^{15}$, and successively determined that SNX13 is indispensable for embryonic development ${ }^{7}$. However, the role of SNX13 in the heart remains unclear. Given that the regulation of RGS proteins is reported to affect the GPCR-mediated signalling and cardiac hypertrophy ${ }^{16}$, SNX13 may have a potential role in the modulation of cardiac function. Our data indicate that SNX13 deficiency causes severe HF. Evidence from ultrastructural and cytochemical assays of SNX13-deficient hearts, which exhibit cellular autolysis and apoptosis, underscore the link between apoptosis and HF. Preventing apoptotic signals by inhibiting caspases largely counteracts the HF phenotype, which further establishes a prominent role for cardiomyocyte apoptosis in HF caused by SNX13 deficiency. The injury of cells or of tissues triggers cellular autolysis, which is the self-digestion or apoptosis of a cell by its own enzymes. Extensive cardiomyocyte autolysis induced by SNX13 deficiency may accelerate the loss of cardiac cells and the progression of cardiac dysfunction. Notably, the mitochondrion-dependent (intrinsic) apoptotic pathway may contribute less to the development of HF in this context; SNX13 reduction does not affect related signal markers ${ }^{17}$, such as cytochrome $c$ release and $\mathrm{Bcl} 2$ translocation, and caspase-9 inhibition confers a weak effect against cardiac dysfunction caused by a SNX13 defect (Fig. 4). Taken together, these findings indicate that SNX13 reduction-mediated HF may be caused primarily by the cardiomyocytes' extrinsic apoptotic pathway. 
a

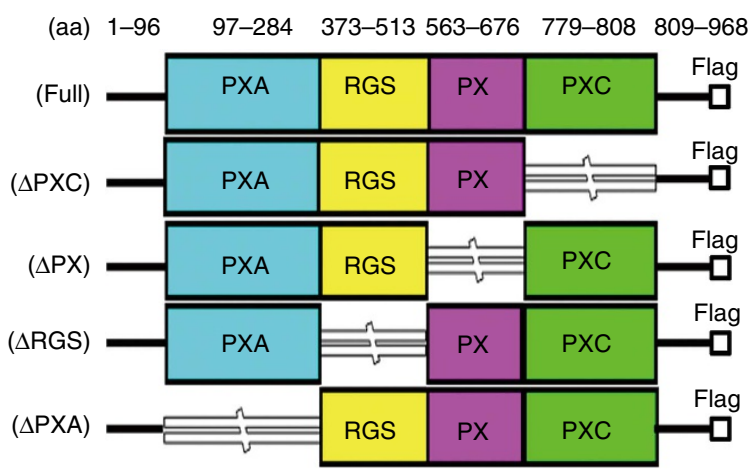

b

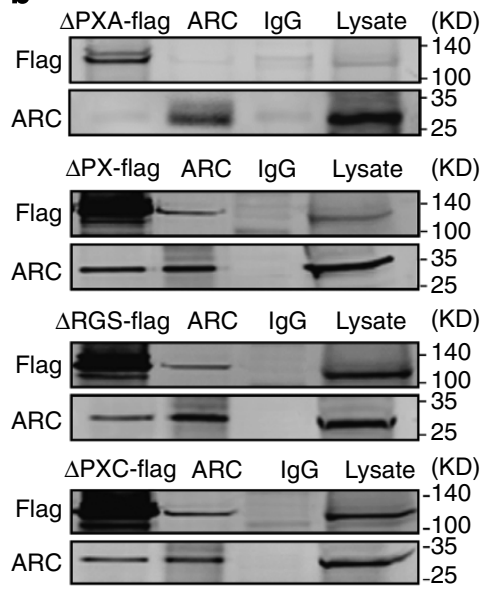

C
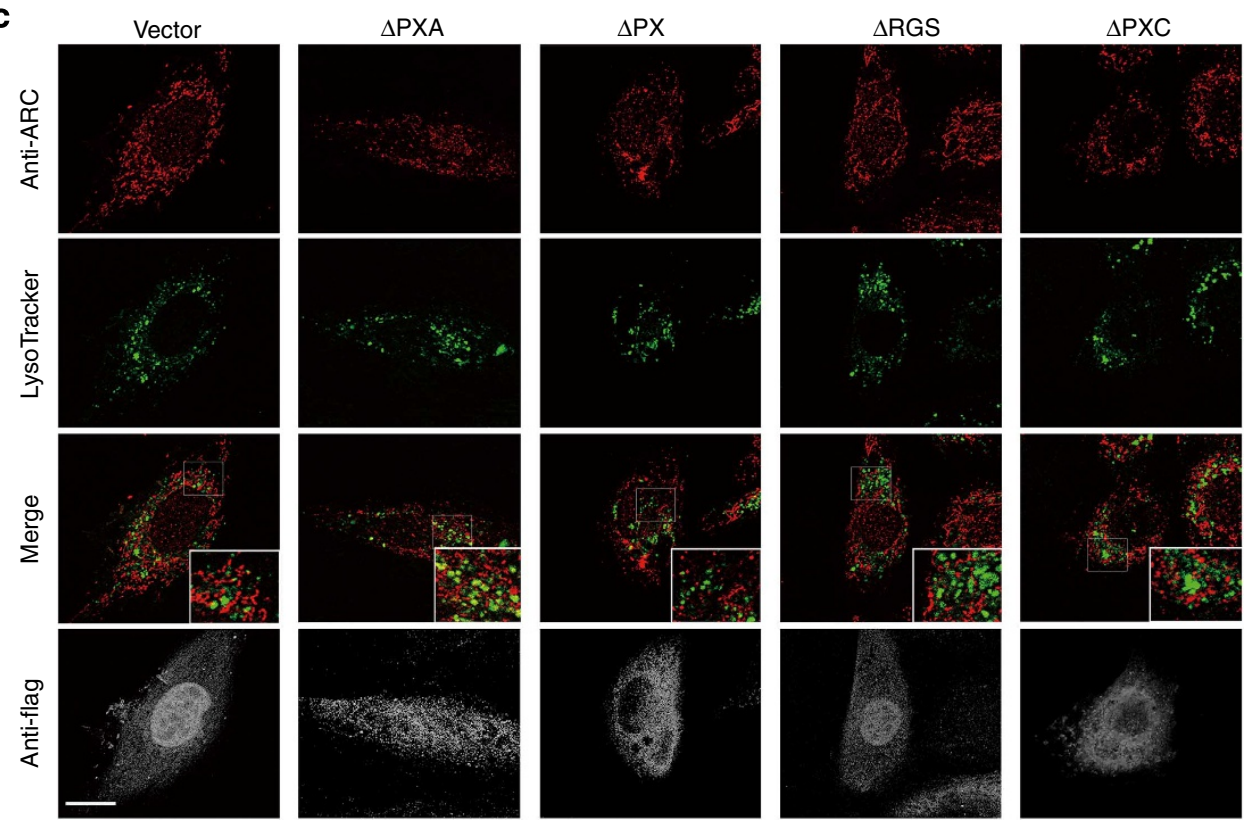

d

e

f
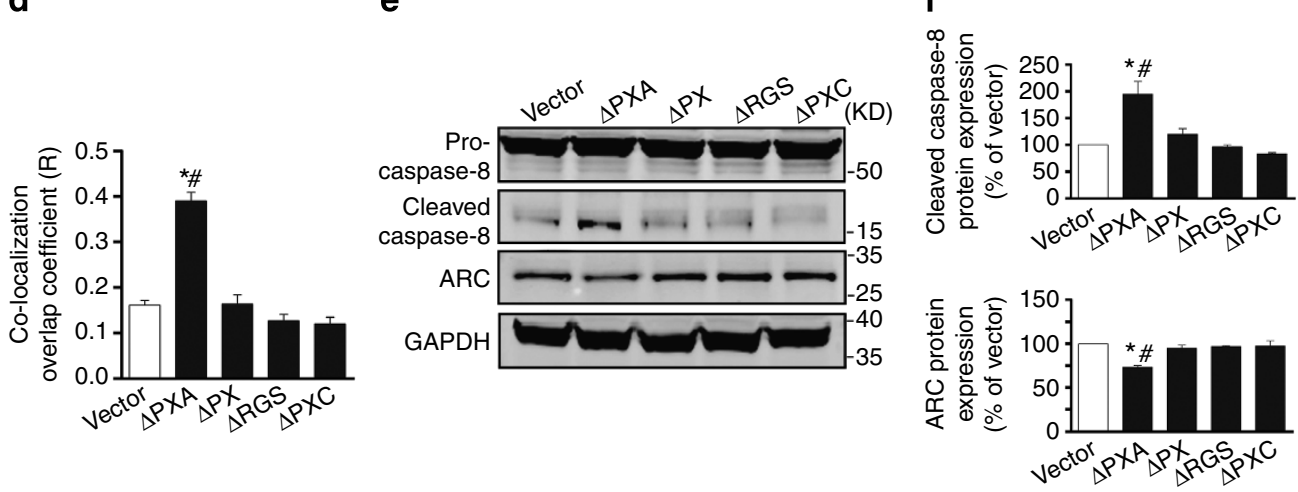

Figure 7 | PXA domain of SNX13 mediates intracellular ARC trafficking. (a) Scheme of the SNX13-truncated proteins. (b) Co-immunoprecipitation of ARC with different SNX13-truncated mutants. (c) Subcellular location imaging of the ARC protein at $48 \mathrm{~h}$ after expressing the SNX13-truncated mutants in $\mathrm{H} 9 \mathrm{C} 2$ cells. Expression of the $\mathrm{N}$-terminal PXA-truncated mutant induced the lysosomal targeting of ARC. The representative images were derived from three to five separate experiments. Scale bar, $20 \mu \mathrm{m}$. (d) Analysis of the co-localization of ARC with lysosomes in cells expressing different SNX13-truncated mutants. Data are means \pm s.e.m. ${ }^{\star} P<0.05$ compared with vector control; $\# P<0.05$ compared with groups other than vector control, by one-way analysis of variance (ANOVA) with Bonferroni's post hoc test. (e) Typical western blot examination of the effects of SNX13-truncated mutants on caspase- 8 activation and ARC expression. (f) Pooled data from e. Data are means \pm s.e.m. ${ }^{\star} P<0.05$ compared with vector control; $\# P<0.05$ compared with groups other than vector control, by one-way ANOVA with Bonferroni's post hoc test. 
One of the principal mechanisms for controlling apoptosis is the regulation of protein-protein interactions ${ }^{18}$. As previously reported, ARC expression in the heart has cardioprotective effects against HF development. This expression blocks the intrinsic and extrinsic apoptotic death pathways by inhibiting caspase activity ${ }^{12}$. In the present study, our findings suggest that SNX13 physically binds to ARC and acts as an important accessory regulator of the interaction between ARC and caspase-8. Reduced SNX13 expression may disrupt ARC sorting, thereby resulting in ARC trafficking to an inappropriate cellular location for degradation, that is, the lysosome. Under these conditions, caspase- 8 is activated due to the removal of ARC-mediated inhibition, leading to apoptotic cardiomyocyte death. The beneficial effects of the restoration of cardiomyocyte ARC expression on the apoptotic death caused by SNX13 reduction further confirmed the crucial role of ARC. Thus, the decreased expression of SNX13 may effectively promote cardiomyocyte apoptosis by inducing ARC degradation, leading to the development of HF.

RGS proteins have been identified as important regulators of cardiovascular physiology ${ }^{16}$. SNX13 also serves as a guanosine triphosphatase-activating protein ${ }^{15}$, which may be implicated in the cardiovascular system. The intracellular cyclic AMP (cAMP) assay showed that SNX13 reduction did not affect the adrenergic receptor activation-mediated cAMP response (Supplementary Fig. 7). Also, H89, which is a specific inhibitor of Gas-mediated cAMP signalling, had no effect on aberrant cardiac function of SNX13 morphants (Supplementary Movie 4). Finally, the SNX13 mutant protein lacking the RGS domain did not affect the endosomal sorting of ARC. Altogether, these results indicate that the SNX13 RGS domain is not responsible for the cardiac dysfunction associated with SNX13 reduction. In addition to the RGS domain, SNX13 possesses three other domains: N-terminal PXA, PX and $\mathrm{PXC}^{5}$. The PX domain is involved in the targeting of proteins to cell membranes in eukaryotes; this targeting is a hallmark feature of the SNX protein family. However, our fragment deletion approach identified that the N-terminal PXA, but not PX and the PXC structure of SNX13, mediated the endosomal sorting of ARC. Thus, the PXA module of SNX13 may be critical for the preservation of ARC recycling in the endosomal system. From a biological perspective, lysosomal turnover primarily contributes to ubiquitinated membrane protein degradation and to proteasomal turnover for cytoplasmic proteins. Several recent studies have reported that the cytoplasmic ARC protein is degraded via the ubiquitinproteasomal pathway ${ }^{19}$ and that an ARC ubiquitin acceptor residue mutation stabilizes and preserves its steady-state levels ${ }^{20}$. Our experiments with a proteasome inhibitor and with lysosome disruption suggested that the ARC protein is degraded through the lysosomal, but not proteasomal, pathway in cells with SNX13 knockdown. Thus, our present study highlights a novel mechanism for the turnover of the ARC protein.

In summary, our findings suggest that a unique signalling mechanism underlies HF induced by SNX13 reduction. A novel aspect of the SNX13-dependent regulatory pathway is the determination of cardiomyocyte fate through modulating ARC turnover. SNX13 deficiency facilitates the lysosomal degradation of ARC, promoting cardiomyocyte apoptosis and HF. The manipulation of SNX13-mediated ARC stability may offer a new therapeutic strategy for HF.

\footnotetext{
Methods

Human heart samples. The investigation complied with the principles that govern the use of human tissues outlined in the Declaration of Helsinki. We have obtained informed consent from patients or close family members.

Left ventricular samples were obtained from five explanted human hearts (Supplementary Table 1). Samples were collected at the time of cardiac
}

transplantation. Three healthy control hearts were obtained from multiorgan donors. The control hearts did not exhibit cardiovascular pathology but were unable to be used for transplantation due to technical reasons.

Animal models. All animal protocols were approved by the Animal Care and Use Committee at the Tongji University School of Medicine and followed the NIH Guidelines for Care and Use of Animals in Research (NIH Publication No. 85-23, revised 1996). HF models were established by transverse aortic constriction in mice. In brief, midline sternotomy was performed on mice that were anaesthetized, intubated and placed on a respirator, and 6.0 Prolene suture was placed around the aorta distal to the brachiocephalic artery. The suture was tightened around a blunt 27 -gauge needle placed close to the aorta. The needle was then removed, and the chest was closed up. Echocardiography was performed at regular intervals to evaluate cardiac function using a 15-Mhz linear-array probe and a Sonos 4500 ultrasonograph (Hewlett-Packard). The animals were killed after 2 months, and the hearts were frozen in liquid nitrogen immediately on death.

Zebrafish husbandry, breeding and functional assessment. A wild-type $A B$ zebrafish line was maintained at $28.5^{\circ} \mathrm{C}$ in $14: 10 \mathrm{~h}$ light/dark conditions, 50 adults per 101 tank. To inhibit pigmentation, $0.003 \% 1$-phenyl-2-thiourea was added to the embryo medium $\left(17 \mathrm{mM} \mathrm{NaCl}, 0.21 \mathrm{mM} \mathrm{KCl}, 0.12 \mathrm{mM} \mathrm{MgSO}_{4}, 0.18 \mathrm{mM}\right.$ $\mathrm{Ca}\left(\mathrm{NO}_{3}\right)_{2}, 1.5 \mathrm{mM}$ HEPES, $\mathrm{pH}$ 7.6). The cardiac output and SV of zebrafish embryos were monitored using a high-speed confocal line-scanning microscope. Briefly, after a 3-min delay to ensure a steady-state level of anaesthesia, images were captured using a Leica SP5 laser-scanning microscope with an Olympus PlanApo $\mathrm{N}(\times 60 / 1.42$ oil) lens using differential interference contrast (DIC) optics. Scan lines were oriented parallel to the flow of blood within the dorsal aorta, just above the yolk ball. Approximately 15,000 lines were acquired per sample at a rate of $0.488 \mathrm{~ms}$ per line allowing a maximum velocity of $4.10 \mathrm{~cm} \mathrm{~s}^{-1}$ to be measured. Using a $20 \mu \mathrm{m}$ line and the optical system described above, a minimum scan rate of $8 \mathrm{~ms}$ per line is required to record the maximum cellular velocity of $0.25 \mathrm{~cm} \mathrm{~s}^{-1}$ observed in these experiments. Heart rates were determined by dividing $60 \mathrm{~s}$ by the average duration of a heartbeat, which was measured by the average peak-to-peak distance of the velocity versus time profile. SV, the volume of blood ejected by the ventricle in one heartbeat, was determined by multiplying the cross sectional area of the aorta by the distance of blood pumped along the aorta in one beat. Cardiac output, the volume of blood ejected by the heart in $1 \mathrm{~min}$, was calculated by multiplying the SV by the heart rate.

Morpholino microinjections. A morpholino-modified oligonucleotide (Gene Tools, Philomath, OR, USA) was directed against the SNX13 translational start site (MO-SNX13: 5'-AGTTTCTCTGACATGCGGCCCATGT-3') and the splice donor sites of exon 2 (MO-SNX13 ${ }^{\text {spl }}$ : $5^{\prime}$-CCAGCCCCTGAATGACGGCACGGAA-3'). The morpholinos (MO-SNX13: $8 \mathrm{ng} \mathrm{nl}^{-1}$; MO-SNX13 ${ }^{\text {spl }}: 4 \mathrm{ng} \mathrm{nl}^{-1}$ ) were injected into wild-type embryos at the one-cell stage. As a negative control, a standard control oligonucleotide ( $5^{\prime}$-AGTATGTCTCACATCCGCCCCATGT- $3^{\prime} ; 8 \mathrm{ng} \mathrm{nl}^{-1}$ ) was injected at the same dose. Injected embryos were allowed to develop at $28.5^{\circ} \mathrm{C}$ until the appropriate developmental stage. Pictures and movies were recorded at the indicated time points after the microinjection treatments.

Transmission electron microscopy. Embryos were fixed in $2 \%$ glutaraldehyde and $2 \%$ paraformaldehyde (PFA) in $0.1 \mathrm{M}$ sodium phosphate buffer (phosphatebuffered saline (PBS); $\mathrm{pH} 7.4$ ) overnight at $4{ }^{\circ} \mathrm{C}$ and post-fixed in $1 \% \mathrm{OsO}_{4}$ in $\mathrm{PBS}$ (pH 7.2). Fixation was followed by staining with $5 \%$ uranyl acetate in $\mathrm{H}_{2} \mathrm{O}$ for $1 \mathrm{~h}$ and then dehydrated by serial steps in ethanol, embedded in Epon and polymerized at $60^{\circ} \mathrm{C}$ for $24 \mathrm{~h}$. Ultrathin sections $(70-90 \mathrm{~nm}$ ) were cut, then stained with uranyl acetate or alternatively stained with lead citrate and uranyl acetate. The sections were analysed under a JEM-1230 transmission electron microscope.

Recombinant adenovirus construction and transfection. For recombinant adenovirus construction, the rat SNX13 complementary DNA (cDNA; Ad-SNX13) and the GFP gene (Ad-GFP; control) cloned by PCR was inserted into pDC315EGFP vector (purchased from Hanbio, Shanghai, China) under the control of the rat cytomegalovirus promoter. The pDC315-SNX13 and pBHGloxE1,3 Cre were co-transfected into HEK293 cells by using LipoFiter transfection reagent (Hanbio) to generate the recombinant adenoviruses. Ad-SNX13 and Ad-GFP were propagated in HEK293 cells. The propagated recombinant adenoviruses in the HEK293 cells were purified and the titre of virus was measured by plaque assays. The stock solutions of Ad-SNX13 and Ad-GFP were $1 \times 10^{10}$ plaque formation unit $\mathrm{ml}^{-1}$. Cardiomyocytes were kept in serum-free medium (control medium) supplemented with transferrin $\left(5 \mathrm{mg} \mathrm{ml}^{-1}\right)$ and insulin $\left(1 \mathrm{mg} \mathrm{ml}^{-1}\right)$ for $24 \mathrm{~h}$ before adenoviral infection.

NRVM isolation and transfection. Single cardiac myocytes from the hearts of 2-3-day-old Sprague Dawley rats were isolated by digestion with collagenase. Briefly, ventricles from neonatal rats were cut into pieces and then dissociated in calcium-free HBSS containing $0.125 \mathrm{mg} \mathrm{ml}^{-1}$ trypsin (Gibco) and $10 \mu \mathrm{g} \mathrm{ml}^{-1}$ DNase II (Sigma). Digestion was performed at $37^{\circ} \mathrm{C}$ in eight to ten 5 -min steps, 
collecting the supernatant to fetal bovine serum (FBS; Gibco) after each step. The collected supernatant was centrifuged and resuspended in DMEM (Gibco) supplemented with $10 \%$ FBS and with $100 \mu \mathrm{M}$ BrdU (5-bromodeoxyuridine; Sigma). The collected cells were passed through a cell strainer $(100 \mathrm{~mm}, \mathrm{BD}$ Falcon $)$ and then seeded onto $100-\mathrm{mm}$ plastic dishes for $2 \mathrm{~h}$ at $37^{\circ} \mathrm{C}$ in $5 \% \mathrm{CO}_{2}$ and humidified atmosphere. The supernatant was then collected and plated onto $1 \%$ gelatin (Sigma)-coated dishes. The next day, the medium was changed by DMEM (Gibco) with 2\% FBS (Gibco), 1\% insulin-transferrin-selenium (ITS) (Gibco) and $100 \mu \mathrm{M}$ BrdU (Sigma). SNX13-siRNA and LAMP1-siRNA were transfected by using Lipofectamine RNAiMAX (Invitrogen). siRNA sequences for SNX13 are as follows: (1) $5^{\prime}$-CAGCCGGUGAUGAUUAUAUTT- $3^{\prime}$ and $5^{\prime}$-AUAUAAUCAUCACC GGCUGTT- $3^{\prime}$; (2) $5^{\prime}$-GAGGCCUUUAUGAACAUUATT- $3^{\prime}$ and $5^{\prime}$-UAAUGUUC AUAAAGGCCUCTT- $3^{\prime}$; and (3) $5^{\prime}$-CCGAGCUUGACAUGUUAAATT- $3^{\prime}$ and $5^{\prime}$-UUUAACAUGUCAAGCUCGGTT- $3^{\prime}$. siRNA sequences specific for LAMP1 is as follows: $5^{\prime}$-GGGUCUACAUGAAGAAUGUTT- $3^{\prime}$ and $5^{\prime}$-ACAUUCUUCAU GUAGACCCTT- $3^{\prime}$. MG132 ( $0.5 \mu \mathrm{M}$, Sigma) or bafilomycin A1 (100 nM, Sigma) was added to culture medium for $48 \mathrm{~h}$ just following SNX13-siRNA transfection.

Mitochondrial protein extraction. For mitochondrial protein analysis, the mitochondria of cardiomyocytes were isolated using mitochondria isolation kit (Sigma). Cross-contamination of cytosol and mitochondria was determined on the basis of the amount of activity of marker enzymes for cytosol (GAPDH) and mitochondria (succinate dehydrogenase). Protein levels in the samples were determined with Bio-Rad protein assay kit.

Immunocytochemistry and immunofluorescence microscopy. Cells were grown at low density on coverslips for indicated time before being processed for immunocytochemistry. They were fixed in $4 \%$ PFA for $15 \mathrm{~min}$ at room temperature, permeabilized in PBS containing $0.01 \%$ Triton X-100 (Sigma) for 10 min and incubated with $1 \%$ bovine serum albumin (BSA) in PBS with Tween 20 (PBST) for 30 min to block unspecific binding of the antibodies. The permeabilized cells were then incubated with primary antibodies (ARC: 1/100, ab126238, Abcam; SNX13:1/ 100, ab67361, Abcam; EEA1:1/300, ab2900, Abcam; Flag: 1/500, F1804, Sigma) for $1 \mathrm{~h}$ at room temperature or overnight at $4{ }^{\circ} \mathrm{C}$ in PBST containing $1 \% \mathrm{BSA}$, and washed three times in PBS. After the cells were incubated for $1 \mathrm{~h}$ at room temperature with secondary antibodies (1/300, Invitrogen) and washed three times in PBS, cells were analysed with a Leica SP5 confocal microscope using a $\times 63$ fluorescence lense. For lysosome labelling, cells were first incubated with $150 \mathrm{nM}$ Lysotracker (Invitrogen) in culture medium for $30 \mathrm{~min}$ at $37^{\circ} \mathrm{C}$. Co-localization was analysed by using ImagePro Plus software.

Histologic sections, H\&E staining and in situ hybridization. Embryos were fixed with $4 \%$ PFA overnight at $4{ }^{\circ} \mathrm{C}$ and mounted in $1 \%$ agarose. The agarose block was gradually dehydrated with $70,90,100 \%$ ethanol and $100 \%$ xylene and embedded in paraffin. The embryos were cut into $7 \mu \mathrm{m}$ sections, which were stained with H\&E. For whole-mount in situ hybridizations, digoxigenin-labelled riboprobes for SNX13 were synthesized from cDNA as run-off transcripts from linearized templates by using the Genius System DIG RNA Labeling Kit. Probes were detected using an alkaline phosphatase-conjugated antibody and visualized with 4-nitroblue tetrazolium/5-bromo-4-chloro-3-indolyl phosphate (NBT/BCIP; Roche Molecular Biochemicals, Indianapolis, IN, USA). Embryos were analysed using a LeicaM205FA microscope.

Detection of cell death. To detect apoptotic cells in live embryos, embryos were dechorionated and soaked in egg water containing $2 \mu \mathrm{g} \mathrm{ml}{ }^{-1} \mathrm{AO}$ at $28^{\circ} \mathrm{C}$ for $30 \mathrm{~min}$. After washing with egg water for eight times, embryos were anaesthetized with tricaine, mounted in $2 \%$ methylcellulose and examined by stereomicroscopy.

To determine whether apoptosis was localized to cardiomyocytes, a transgenic zebrafish line expressing the cardiac-specific light myosin 2 ( $\mathrm{cmlc} 2$ ) was employed. The transgenic embryos were further used for the analysis of apoptosis by wholemount immunofluorescence to detect activated caspase-3. Briefly, zebrafish embryos were collected in a Petri dish in $1 \times$ egg water. Then, up to 40 embryos were transferred to a $1.5-\mathrm{ml}$ tube and rinsed two times with $1 \mathrm{ml}$ of $1 \times$ PBST. After removing PBST, $1 \mathrm{ml}$ of $4 \%$ PFA was added and the tubes were placed on a gentle rocker at $4{ }^{\circ} \mathrm{C}$ overnight. Following the fixation, embryos were treated with $100 \%$ ice-cold methanol $(1 \mathrm{ml})$ at $-20^{\circ} \mathrm{C}$ for $2 \mathrm{~h}$ and subsequently washed with fresh PDT $(1 \times$ PBST, $1 \%$ DMSO, $0.3 \%$ Triton-X) for 30 min. Finally, $1 \mu \mathrm{l}$ rabbit anti-activated caspase-3 antibody (1:100, C8487, Sigma) was added with the appropriate dilution of an anti-rabbit fluorophore-conjugated antibody that absorbs blue light and emits green light. After addition of the secondary antibody, embryos were kept in dark for analysis.

Reverse transcription-PCR. Heart, liver, muscle and brain tissues were separated from adult zebrafish, and mRNA was extracted from the pooled tissue using TRIzol reagent (Invitrogen). For the subsequent PCR, the following protocol was used: $4 \mu \mathrm{l}$ buffer, $2 \mu \mathrm{l} \mathrm{MgCl} 2,1 \mu \mathrm{l}$ deoxy-ribonucleoside triphosphate (dNTP) $\left(10 \mathrm{mmoll}^{-1}\right)$, $1 \mu \mathrm{l}$ of each primer $\left(20 \mu \mathrm{moll}^{-1}\right), 8 \mu \mathrm{lDNA}, 0.5 \mu \mathrm{l}$ (2.5 U) AmpliTaq Gold polymerase and $32.5 \mu \mathrm{l} \mathrm{H} \mathrm{H}_{2} \mathrm{O}$. PCR was performed at $94{ }^{\circ} \mathrm{C}$ for $2.45 \mathrm{~min}$, followed by
63 cycles at $94{ }^{\circ} \mathrm{C}$ for $0.45 \mathrm{~min}, 57^{\circ} \mathrm{C}$ for $0.45 \mathrm{~min}$ and $72^{\circ} \mathrm{C}$ for $0.45 \mathrm{~min}$. The reverse transcription-PCR primer sequences were as follows: DaSNX13-RT-F (5'-GTTGAAGCACATCATCGGCG-3') and DaSNX13-RT-R (5'-GGAACAGCG TCTCCAGGAAG- $3^{\prime}$ ). After a final extension of $72^{\circ} \mathrm{C}$ for $7 \mathrm{~min}$, the PCR products were electrophoresed on a $1.8 \%$ agarose gel and ethidium bromide stained.

Western blot analysis. Ventricular tissue or myocytes were lysed in radioimmunoprecipitation buffer $(50 \mathrm{mM}$ Tris, $150 \mathrm{mM} \mathrm{NaCl}, 1 \mathrm{mM} \mathrm{Na}$-EDTA, $1 \%$ Triton X-100, $1 \%$ sodium deoxycholate, $2 \mathrm{mM} \mathrm{NaF}$ and $200 \mu \mathrm{M} \mathrm{Na}_{3} \mathrm{VO}_{4}$ at $\mathrm{pH}$ 7.4). The lysates were heated to $95^{\circ} \mathrm{C}$, size fractionated in NuPAGE gels (Invitrogen) and transferred to polyvinylidene fluoride membranes. The membranes were blocked with $5 \%$ skim milk for $1 \mathrm{~h}$ and incubated overnight at $4{ }^{\circ} \mathrm{C}$ with the appropriate primary antibodies: SNX13 (1:1,000, AP12244b, Abgent), ARC (1:1,000, NBP1-76952, Imgenex), GAPDH (1:1,000, AG019, Beyotime), caspase 8 (1:1,000, AC056, Beyotime), caspase 9 (1:1,000, AC062, Beyotime), caspase 3 (1:1,000, AC031, Beyotime), Bcl-2 (1:1,000, AB112, Beyotime), Bax $(1: 1,000,5,023$, Cell Signaling), cytochrome $c(1: 1,000,11,940$, Cell Signaling), COXIV (1:1,000, AC610, Beyotime), LAMP1 (1:1,000, ab13525, Abcam), Rab5 (1:1,000, Cell Signaling), EEA1 (1:1,000, ab2900, Sigma), $\beta$-actin (1:1,000, AA128, Beyotime) and flag (1:1,000, F1804, Sigma). After incubation with horseradish peroxidase-conjugated secondary antibodies for $1 \mathrm{~h}$ at room temperature, the immunoblots were visualized using enhanced chemiluminescence (Pierce). Band intensities were analysed using Quantity One image software (Bio-Rad). Uncropped scanned images of western blots are presented in Supplementary Fig. 8.

Flow cytometry. After transfecting for $48 \mathrm{~h}$, the myocytes were stained with Annexin V PE and 7-aminoactinomycin D (7-AAD) (Annexin V PE Apoptosis Detection Kit PE, eBioscience) and analysed using flow cytometry (Beckman Coulter). The data were expressed as percentages of the total cell population.

Immunoisolation of Rab5-positive endosomes. NRVMs were washed with PBS at $4{ }^{\circ} \mathrm{C}$ and suspended in homogenization buffer $(3 \mathrm{mM}$ imidazole, $250 \mathrm{mM}$ sucrose, $0.5 \mathrm{mM}$ EDTA and complete EDTA-free protease inhibitor cocktail; Roche Diagnostics, IN). Cells were homogenized and centrifuged for $10 \mathrm{~min}$ at 3,000 r.p.m. The resulting postnuclear supernatant was adjusted to $40.6 \%$ sucrose, placed in 14-ml capacity ultra-clear centrifuge tubes (Beckman), overlaid with $6 \mathrm{ml}$ of $35 \%$ sucrose and $4 \mathrm{ml}$ of $25 \%$ sucrose and then centrifuged in a SW $41 \mathrm{Ti}$ rotor at $108,000 \mathrm{~g}$ for $3 \mathrm{~h}$ at $4{ }^{\circ} \mathrm{C}$. The endosome-enriched fraction at the $25-35 \%$ sucrose interface was collected. Rabbit anti-Rab5 (1:50, 3,547, Cell Signaling) or a nonspecific rabbit IgG (Immunoglobulin G; 1:50, I5006, Sigma) was added and incubated with the isolated endosomes overnight at $4{ }^{\circ} \mathrm{C}$ with rotation. In addition, $50 \mu \mathrm{l}$ Dynabeads (Invitrogen) were washed three times and incubated with $1 \mathrm{ml}$ PBS containing $1 \%$ BSA overnight at $4{ }^{\circ} \mathrm{C}$. The following day, the beads were recovered, added and incubated with Rab5 antibody or IgG-endosome fractions for $6 \mathrm{~h}$ at $4{ }^{\circ} \mathrm{C}$ with rotation. The bead-antibody-endosome complexes were collected and washed twice. Then, the samples were analysed by $10 \%$ SDS-PAGE and immunoblotting.

Immunoprecipitation analysis. Adult rat ventricles were cut into small pieces and homogenated in lysis buffer. After centrifugation, the supernatants were collected and incubated overnight at $4{ }^{\circ} \mathrm{C}$ with primary antibodies SNX13 (1:50, AP12244b, Abgent) and ARC (1:50, NBP 1-76952, Imgenex) by constant stirring. In addition, $50 \mu$ Dynabeads (Invitrogen) were incubated with $1 \mathrm{ml} \mathrm{1 \%}$ BSA/PBS overnight at $4{ }^{\circ} \mathrm{C}$. The following day, the beads were recovered, added and incubated with the antibodies for $6 \mathrm{~h}$ at $4^{\circ} \mathrm{C}$ with rotation. The bead-antibody complexes were collected and washed twice. Then, the samples were analysed by $10 \%$ SDS-PAGE and immunoblotting.

DNA ladder analysis. To detect DNA fragmentation, genomic DNA was isolated from NRVMs using a DNA Isolation Kit (Puregene) and resolved on a $1.5 \%$ agarose gel.

DNA construction. For expression of recombinant proteins in mammalian cells, different truncated fragments of human SNX13 were subcloned into the expression vector Flag (Clontech, Palo Alto, CA, USA). cDNAs encoding SNX13 fragments were derived by PCR. These constructs were transformed into Escherichia coli DH5 $\alpha$ cells (Tiangen, Shanghai, China) and were screened by PCR with the corresponding primers for each construct. Positive colonies were selected, and the correct inserts were confirmed by DNA sequencing. DNA constructions were transfected into isolated myocytes by using Lipofectamine 2000 (Invitrogen) and analysed by $10 \%$ SDS-PAGE and immunoblotting. Cell lysates were collected for immunoprecipitation analysis by using primary antibodies flag (1:50, F1804, Sigma) and ARC (1:50, NBP 1-76952, Imgenex).

Fluorescence resonance energy transfer measurement. Neonatal rat cardiomyocytes transfected by PKA biosensor ICUE1 (provided by Dr Jin Zhang, Johns Hopkins University School of Medicine) were rinsed and maintained in PBS with 
calcium for fluorescence resonance energy transfer recording. Cells were imaged on a Zeiss Axiovert $200 \mathrm{M}$ microscope with a cooled charge-coupled device camera. Dual-emission ratio imaging was acquired with a 420DF20 excitation filter, a 450DRLP dichroic mirror and two emission filters (475DF40 for cyan and 535DF25 for yellow). Exposure time was $200 \mathrm{~ms}$, and images were taken every $8 \mathrm{~s}$. Images in both channels were subjected to background subtraction, and ratios of cyan-to-yellow colour were calculated at different time points.

cAMP assay. Cardiomyocytes' intercellular cAMP concentration was detected by using cAMP-Glo Assay kit (Promega). Briefly, after transfection with SNX13siRNA or scramble for $48 \mathrm{~h}$, cardiomyocytes were trypsinized, washed and distributed equally to a 96 -well assay plate with white clear bottom. On day 2 , medium was changed with $1 \mu \mathrm{M}$ isoproterenol induction buffer for $30 \mathrm{~min}$, and then the cell intercellular cAMP concentration was measured according to the assay kit protocol. The cAMP accumulation after isoproterenol treatment of the SNX13-siRNA group was compared with the control group.

Measurement of proteasomal activity. Proteasome activity was analysed by Proteasome-Glo Chymotrypsin-Like Cell-based Assay Kit (Promega). Briefly, NRVMs were treated with 0.5 or $1 \mathrm{mM} \mathrm{MG132}$ for $48 \mathrm{~h}$, and then cells were trypsinized, washed twice by PBS and counted to optimize cell number for a 96-well plate. The proteasome activity was measured following the assay kit protocol.

Statistical analysis. All data are expressed as the mean \pm s.e.m. In all experiments, $N$ represents the number of animals, and $n$ represents the number of experiments. An unpaired $t$-test with Welch's correction or a one-way analysis of variance is used to compare experimental groups with their appropriate control unless otherwise specified; $P<0.05$ is considered significant for all experiments.

\section{References}

1. Roger, V. L. et al. Heart disease and stroke statistics-2012 update: a report from the American Heart Association. Circulation 125, e2-e220 (2012).

2. Kehat, I. \& Molkentin, J. D. Molecular pathways underlying cardiac remodeling during pathophysiological stimulation. Circulation 122, 2727-2735 (2010).

3. von Zastrow, M. \& Sorkin, A. Signaling on the endocytic pathway. Curr. Opin. Cell Biol. 19, 436-445 (2007).

4. Ellson, C. D., Andrews, S., Stephens, L. R. \& Hawkins, P. T. The PX domain: a new phosphoinositide-binding module. J. Cell Sci. 115, 1099-1105 (2002).

5. Cullen, P. J. Endosomal sorting and signalling: an emerging role for sorting nexins. Nat. Rev. Mol. Cell Biol. 9, 574-582 (2008).

6. Teasdale, R. D. \& Collins, B. M. Insights into the PX (phox-homology) domain and SNX (sorting nexin) protein families: structures, functions and roles in disease. Biochem. J. 441, 39-59 (2012).

7. Zheng, B. et al. Essential role of RGS-PX1/sorting nexin 13 in mouse development and regulation of endocytosis dynamics. Proc. Natl Acad. Sci. USA 103, 16776-16781 (2006)

8. Bakkers, J. Zebrafish as a model to study cardiac development and human cardiac disease. Cardiovasc. Res. 91, 279-288 (2011).

9. Communal, C. et al. Functional consequences of caspase activation in cardiac myocytes. Proc. Natl Acad. Sci. USA 99, 6252-6256 (2002).

10. Heikaus, S., Kempf, T., Mahotka, C., Gabbert, H. E. \& Ramp, U. Caspase-8 and its inhibitors in RCCs in vivo: the prominent role of ARC. Apoptosis 13, 938-949 (2008).

11. Jo, D. G. et al. Calcium binding of ARC mediates regulation of caspase 8 and cell death. Mol. Cell. Biol. 24, 9763-9770 (2004).
12. Donath, S. et al. Apoptosis repressor with caspase recruitment domain is required for cardioprotection in response to biomechanical and ischemic stress. Circulation 113, 1203-1212 (2006).

13. Long, X. et al. Enhanced expression of p53 and apoptosis induced by blockade of the vacuolar proton ATPase in cardiomyocytes. J. Clin. Invest. 101, 1453-1461 (1998).

14. Dreger, H. et al. Nrf2-dependent upregulation of antioxidative enzymes: a novel pathway for proteasome inhibitor-mediated cardioprotection. Cardiovasc. Res 83, 354-361 (2009).

15. Zheng, B. et al. RGS-PX1, a GAP for GalphaS and sorting nexin in vesicular trafficking. Science 294, 1939-1942 (2001).

16. Riddle, E. L., Schwartzman, R. A., Bond, M. \& Insel, P. A. Multi-tasking RGS proteins in the heart: the next therapeutic target. Circ. Res. 96, 401-411 (2005).

17. Helen, M. B. Stressed to death: regulation of apoptotic signaling pathways by the heat shock proteins. Sci. STKE 93, re1 (2001).

18. MacLellan, W. R. \& Schneider, M. D. Death by design. programmed cell death in cardiovascular biology and disease. Circ. Res. 81, 137-144 (1997).

19. Foo, R. S., Chan, L. K., Kitsis, R. N. \& Bennett, M. R. Ubiquitination and degradation of the anti-apoptotic protein ARC by MDM2. J. Biol. Chem. 282, 5529-5535 (2007).

20. Nam, Y. J. et al. The apoptosis inhibitor ARC undergoes ubiquitinproteasomal-mediated degradation in response to death stimuli: identification of a degradation-resistant mutant. J. Biol. Chem. 282, 5522-5528 (2007).

\section{Acknowledgements}

We thank the Electron Microscopy Facility of the Institute of Neuroscience, Shanghai Institutes for Biological Sciences of Chinese Academy of Sciences, and Dr Yu Kong and Liangliang $\mathrm{Wu}$ in particular for assistance with electron microscopy experiments. This work was supported by the National Key Basic Research Program of China (2013CB531100, to Y.-H.C.), the Foundation for Innovative Research Groups of the National Natural Science Foundation of China (81221001, to Y.-H.C.), the Major International Joint Research Program Fund of China (81120108004, to Y.-H.C.), the National Natural Science Foundation of China (81170224 and 81270313, to J.L.; 31271214 , to Y.-H.C.) and the Shanghai Natural Science Fund (10JC1414700, to Y.-H.C.).

\section{Author contributions}

Y.-H.C. and J.L. conceived and designed this study. J.L., D.Z., C.L., D.S., M.Q., J.F., T.Y., X.X., D.L., L.X., H.Z., Y.L., J.C., J.Y., W.J., Y.C., Y.Z. and L.P. performed the experiments. J.L., D.Z., L.P., Z.Z. and Y.-H.C. analysed the data. J.L., L.P., Z.Z. and Y.-H.C. provided discussions and advice. Y.-H.C. and J.L. wrote the manuscript. Y.-H.C. supervised the project.

\section{Additional information}

Supplementary Information accompanies this paper at http://www.nature.com/ naturecommunications

Competing financial interests: The authors declare no competing financial interests.

Reprints and permission information is available online at http://npg.nature.com/ reprintsandpermissions/

How to cite this article: Li, J. et al. SNX13 reduction mediates heart failure through degradative sorting of apoptosis repressor with caspase recruitment domain. Nat. Commun. 5:5177 doi: 10.1038/ncomms6177 (2014). 